\title{
Los nevados del Aconquija como sitio de frontera Y ESPACIO DE OBSERVACIÓN LUNAR, TuCUMÁN, NOROESTE de Argentina
}

\author{
Ricardo Moyano', Martín Gustavo Díaz
}

\section{* Introducción}

Resumen

Se presentan los aspectos sociales vinculados al concepto de frontera, el ushnu y la observación lunar en el sitio inca de La Ciudacita, provincia de Tucumán, noroeste de Argentina. El análisis se centra en la posibilidad de observar el lunisticio o parada mayor al sur, en relación a conceptos como la latitud geográfica, la arquitectura y los marcadores de horizonte desde la perspectiva de la astronomía cultural. Como hipótesis se presenta al ushnu como la representación lunar del cenit al sur del trópico de Capricornio, subrayando la utilidad de este rasgo arquitectónico para el seguimiento y predicción de eclipses. Los resultados señalan una estrecha relación entre el ushnu, el gnomon y la observación de las fases lunares, posiblemente vinculada con las relaciones de poder y la expansión del Tawantinsuyu cerca del año 1470 DC.

Palabras claves: astronomía lunar - ushnu - Collasuyu - Tucumán La Ciudacita.

Abstract

We present social aspects related to the concepts of border, ushnu and lunar observation at the inca site of La Ciudacita, Tucuman province, northwestern Argentina. The analysis focused on the possibility of observing the lunistice or major lunar standstill, in relation to concepts of geographical latitude, architecture and horizon landmarks from the perspective of cultural astronomy. Our hypothesis is that the ushnu serves as a representation of the Moon at the zenith, south of the Tropic of Capricorn, highlighting its utility for following and predicting eclipses. Results show that the relationship between the ushnu and gnomon in observing the Moon's phases was possibly linked to relations of power and the expansion of Tawantinsuyu, circa $1470 \mathrm{AD}$.

Key words: lunar astronomy - ushnu - Collasuyu - Tucumán La Ciudacita.

Recibido: Octubre 2013. Aceptado: Marzo 2015
La astronomía cultural como interdisciplina intenta responder a las inquietudes provenientes de la relación que establece el ser humano con su entorno, particularmente el cielo como una categoría social dentro de la antropología (Iwaniszewski 2011). En lo que refiere al pasado de sociedades no occidentales, la arqueoastronomía, como subdisciplina de la astronomía cultural, se centra en el estudio de los antiguos sistemas astronómicos y calendáricos partiendo de la premisa que éstos son el reflejo de la cultura, por ende resultado de categorías espaciotemporales dentro de la noción de "mundo de la vida" o cosmovisión (Iwaniszewski 2011; Moyano 2011, 2013; Moyano y Uribe 2012).

Según Tilley (1994), espacio es un concepto más abstracto que el de lugar, por cuanto este último se convierte en un centro de significación a escala humana, que tiene su representación en el día a día. Razón por la cual, el significado de lugar tiene un sentido existencial dentro del contexto de la experiencia humana, un punto de referencia que distingue significados y valores, y también como un reservorio de la memoria. Un lugar tiene cualidades metonímicas y experimenta diferentes densidades dependiendo de quién lo experimenta, razón por la cual crea y mantiene una identidad particular, siempre haciendo referencia a elementos relacionales y concretos de la cultura (Tilley 1994: 18).

Dentro de la astronomía - a simple vista - toma especial importancia la definición del lugar o punto de observación (Aveni 2005), como "aquellos centros de

1 UNAM, Instituto de Investigaciones Históricas. Circuito Mario de la Cueva S/Nº Coyoacán, Ciudad Universitaria, O4510 Ciudad de México, D.F., MÉXICO.Email: mundosubterraneo2@yahoo.es

2 Laboratorio de Geoarqueología, Facultad de Ciencias Naturales e Instituto Miguel Lillo. Universidad Nacional de Tucumán. Miguel Lillo 205, 4000 San Miguel de Tucumán, ARGENTINA. Email:el_maiten@hotmail.com 
significado humano... [que]... proveen seguridad ontológica, es decir, el punto de partida para la definición de los conceptos de lugar-instante, el yo y el otro, la causalidad y la relación [que entregan]... una estructura y una historia, dentro de una práctica social discursiva" (Moyano 2010a: 74). Dicho de otra manera, el punto de observación se entiende como el lugar que ocupa un observador por tiempo prolongado y permanente, que mira al horizonte y al cielo haciendo uso de determinadas marcas, p. e. un cerro, como referentes del movimiento de distintos objetos celestes. De este modo, este lugar adquiere no sólo un sentido práctico (Giddens 1995), sino también discursivo dentro de acciones sociales recurrentes como solsticios, equinoccios, lunisticios y eclipses vinculados, posiblemente, con momentos de importancia ritual dentro de un sistema de calendario (Iwaniszewski 2011; Moyano 2013, siguiendo a Hardman y Hardman 1992).

De esta manera, el ushnu inca definido como un punto infinitesimal en el espacio (Moyano 2010a, 2010b, retomando a otros autores) y que arquitectónicamente incluyó a la plataforma (con o sin escalera), gnomon, agujero (cocha) y/o sistema de irrigación, habría permitido no sólo ejercer la política, la administración y la religión en el Cuzco y principales centros incas del Tawantinsuyu (Gasparini y Margolies 1977; Raffino et al. 1997; Raffino 1981, 2004; Hyslop 1990; Farrington 2014). Este lugar también pudo funcionar como un observatorio astronómico para solsticios, equinoccios, pasos cenitales, lunisticios y estrellas (Pino 2004, 2005; Moyano 2010b; Jacob et al. 2011, 2013), el cual se encontraba relacionado, al menos en el Cuzco, con las fechas de siembra y cosecha (agosto y abril), la fiesta de la Citua (mes lunar de septiembre) y los rituales propiciatorios vinculados con la acción de dar de beber a la pacha (Zuidema 1980, 1989, 2011; Meddens 1997).

En este contexto, Ianiszewski (2010) y Farrington (Com. personal 2012 - 2013) plantearon la posibilidad de que la luna, por efecto de la diferencia que tiene el ángulo de su órbita con respecto a la eclíptica, igual a $5^{\circ} 09^{\prime}$ promedio, tuviera alguna incidencia en la ubicación de sitios incas al sur del trópico de Capricornio. Para Moyano (2013), ésto habría permitido no sólo registrar el ciclo metónico a partir de la posición de la Luna en el cenit cada 19 años, sino también conocer el momento de ocurrencia y repetición de eclipses lunares, conceptualizado como un momento fatídico para los incas, gracias a la observación de la luna llena en parada mayor o menor, junto con el crossover del equinoccio de primavera.

El crossover se define como el momento del año cuando la luna, en fase llena, pasa de un cuadrante del cielo al otro, en sentido inverso al movimiento que tiene el sol, en el horizonte del oriente. Recibe también el nombre de equinoccio megalítico y se relaciona con la aparición de la luna llena del equinoccio de marzo en el hemisferio norte, $7^{\circ}$ a $9^{\circ}$ al sur de la línea este-oeste. Por su importancia astronómica se vincula con las fechas de cambio de estación, dando inicio a la primavera tras el invierno con acimuts con promedios calculados de $97,3^{\circ}$ en 110 años, lo que corrige el movimiento de la regresión de los nodos (da Silva 2004, 2010). Para el hemisferio sur, la lógica funciona a la inversa, posibilitando el hecho que orientaciones al norte del este $\left(90^{\circ}\right)$, puedan estar marcado la posición de la luna llena cercana al equinoccio de septiembre, es decir, el inicio de la primavera (Bustamante y Moyano 2013; Moyano 2013).

En el caso del sitio La Ciudacita, provincia de Tucumán, Argentina (27 10'52.4"S, 66 $00^{\circ} 24.5^{\prime \prime} \mathrm{W}, 4384 \mathrm{msnm}$ ) (Figura 1), uno de los complejos arqueológicos de alta montaña mejor conservados del Collasuyu, se tienen indicios desde finales del siglo XIX de la presencia de un ushnu, grandes plazas, gnomon (piedra equinoccial), cerámica y arquitectura inca. Esto despertó el interés de muchos arqueólogos y grupos de investigación (Mansfeld 1948; Paulotti 1959, 1967; Beorchia 1985, 2001; Hyslop y Schobinger 1991; Bravo 1993; Lazarovich 1996; Martel et al. 2002; Ataliva et al. 2010), que dieron cuenta detallada de la monumentalidad arquitectónica del lugar. Desde la arqueoastronomía los trabajos de Orlando Bravo (Bravo 1993; Beorchia 2001) plantearon la posibilidad del manejo de un calendario en base a solsticios y equinoccios, con interesantes hipótesis provenientes de la física y el cálculo geodésico.

Intentamos ir un poco más allá y vislumbrar la posibilidad de entender al sitio arqueológico de La Ciudacita desde la perspectiva interdisciplinaria, para lo cual incluimos un análisis de conceptos provenientes de la astronomía cultural, particularmente la variable lunar. Se incluye también la noción de frontera -en su sentido amplio- para explicar la existencia del ushnu y otros 


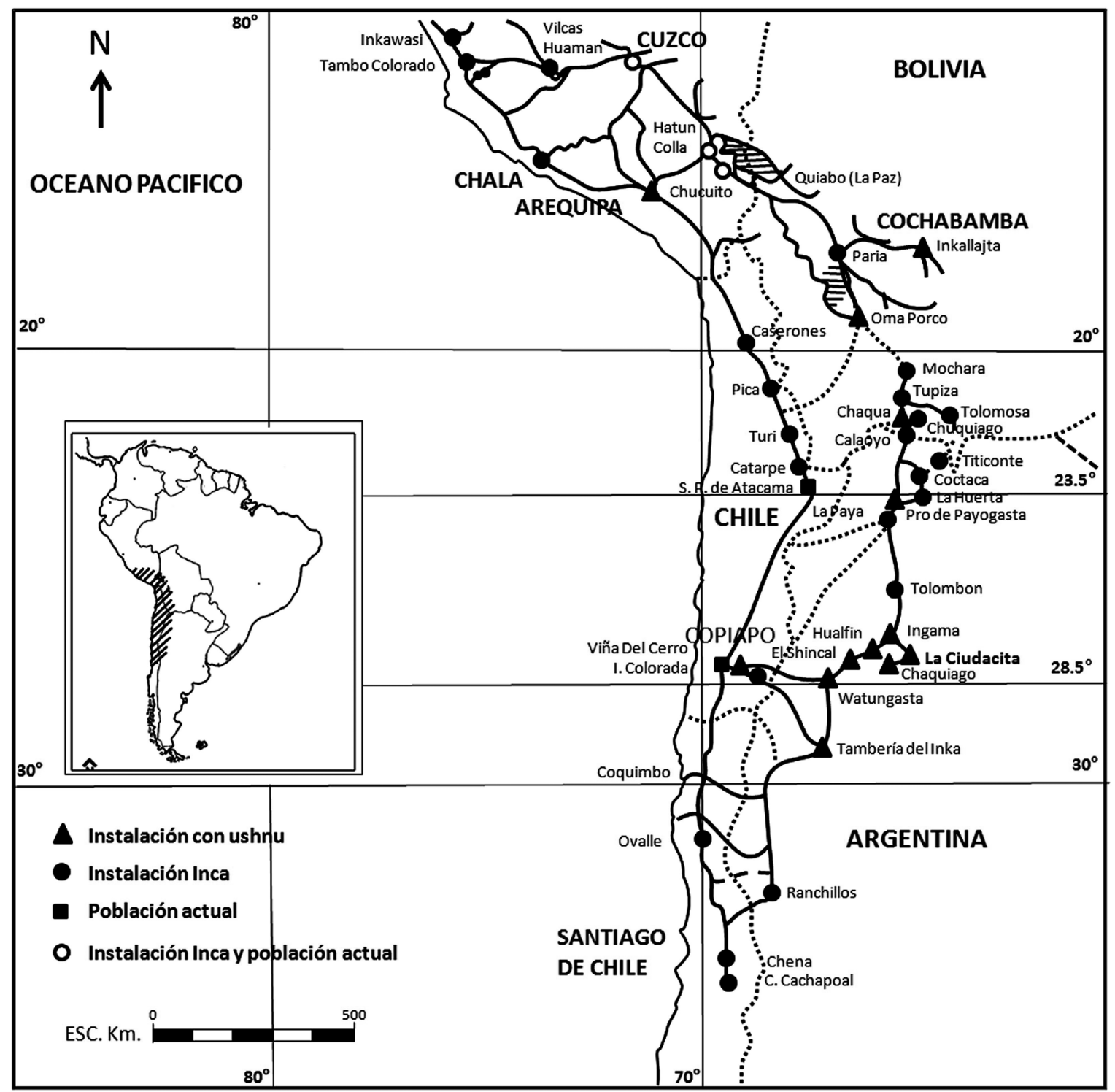

Figura 1. Mapa de ubicación general (modificado de Raffino et al. 1997:22).

instrumentos de observación astronómica, como respuestas y herramientas de coerción ideológica dentro de la política expansiva de los incas, a partir de un sistema local de ceques y sus implicancias sociales en el territorio (Moyano y Díaz 2013).

La metodología de investigación incluyó la observación y registro de salidas y puestas de sol desde el sitio arqueológico La Ciudacita (ushnu y piedra equinoccial). En el trabajo de campo se utilizó un tránsito mecánico, así como una brújula magnética, para el registro de ángulos verticales y horizontales ${ }^{3}$. La investigación contempló el uso de fotografías y cartografía digital para el cálculo de cuencas y líneas visuales, además de la identificación de topónimos y elementos característicos del paisaje. El registro visual se llevó a cabo gracias a la fotografía digital y la reconstrucción del horizonte observado, con la ayuda del programa Photoshop. Con GPS se geo-referenció el sitio con el fin de obtener la posición exacta del lugar en coordenadas geográficas (latitud/longitud), además

3 En el caso de las medidas tomada con brújula, todas fueron corregidas con ayuda de una calculadora de declinación magnética: http:// www.ngdc.noaa.gov/geomag-web/ 
de la altura con respecto al nivel del mar (Datum WGS 84). El cálculo para la declinación del Sol y la Luna con respecto a marcadores del paisaje se realizó gracias a un sistema de referencia de horizonte ${ }^{4}$. El análisis astronómico de fechas y declinaciones se llevó a cabo con el software Starcalc 5.72, tomando en cuenta siempre el factor de paralaje $e^{5}$ refracción atmosférica ${ }^{6}$ (Sprajc 2010), junto con los datos entregados por Astronomical Almanac (USA) e IGiK (Polonia) ${ }^{7}$. Para el análisis final se elaboró una montea luni-solar siguiendo el modelo de Stuven (1972) y Beltrán de Quintana (1982) ${ }^{8}$. En términos de logística, se tomó en cuenta factores como la distancia al lugar de observación (tiempo de caminata), estabilidad atmosférica (día soleado), fechas de observación (inicios de invierno), naturaleza de los horizontes, condiciones de conservación (muros y estructuras) y acceso (permisos provinciales y comunidad local).

Como hipótesis planteamos que La Ciudacita, como producto cultural, tuvo la posibilidad de establecer un límite físico e ideológico entre los grupos incanizados y las poblaciones de yunga, y también permitió manejar ciertos ciclos astronómicos y calendáricos útiles para las relaciones productivas y comerciales en un espacio de frontera. Ello, a partir del reconocimiento de todos aquellos elementos sagrados en la topografía y el cielo, como fueron

4 Fórmulas proporcionadas y modificadas por S. Iwaniszewski (2008-2013).

5 El paralaje corresponde a la desviación angular de la posición aparente de un objeto, dependiendo el punto de vista elegido (Hawkins 1966). Formula: $\mathrm{h}=($ hteo $-\mathrm{r})+\mathrm{p}$, donde: $\mathrm{h}=$ altura del horizonte corregida, hteo $=$ altura medida con teodolito, $r=$ refracción y $\mathrm{p}=$ paralaje ( $\left.57^{\prime}\right)$.

6 Distorsión producida por la atmósfera de la posición real de un objeto en el cielo, cuya luz tiende a ser perpendicular a la superficie del globo en el punto donde se sitúa un observador. Cuanto más bajo esté el objeto en el horizonte, más distorsionada será la trayectoria de su luz; como consecuencia, éste se verá en una posición algo más elevada de la que realmente se encuentra. El efecto de la refracción atmosférica es mayor en latitudes altas y también con objetos que salen y se ponen cerca de los polos (Aveni 2005: 144-147).

7 http://www.igik.edu.pl/index.php/pl/rocznik-astronomiczny

8 http://www.jaloxa.eu/resources/daylighting/sunpath.shtml

9 Según un relato de 1951 de don Augusto Escudero, propietario de la estancia El Tesoro de la pirca del Tipillas, mineros chilenos en la década de 1920 extrajeron un "muñequito de $15 \mathrm{~cm}$ de altura, con ponchito de colores, mascara de oro y coronamiento de plumas" (Bravo 1993: 12). los cerros, el Sol y la Luna, entre otros, como base de un sistema mnemotécnico destinado a sostener y manejar las relaciones de poder entre los incas, las elites locales y las poblaciones del Tucumán.

\section{La Ciudacita}

La Ciudacita es un sitio inca de alta montaña ubicado en la vertiente oriental de los Nevados de Aconquija, a una altura aproximada de $4384 \mathrm{msnm}$, sobre uno de los contrafuertes del cerro Tipillas de los Cerritos (5420 msnm) $)^{9}$, en un filo orientado NW-SE que baja desde el Morro de las Ruinas o cerro Las Cuevas (4900 msnm) ${ }^{10}$. El sitio se compone de dos sectores claramente diferenciados: Pueblo Viejo Arriba (Corrales) y Pueblo Viejo Abajo (Calasasaya), unidos por una calzada empedrada y distinguible conocida como "camino del Inca", todo flanqueado por dos grandes quebradas abruptas: río Jaya al este y el río Las Pavas al norte, tributarios del río Gastona, dominando gran parte de la llanura tucumana (Paulotti 1959, 1967; Bravo 1993; Beorchia 2001) (Figura 2).

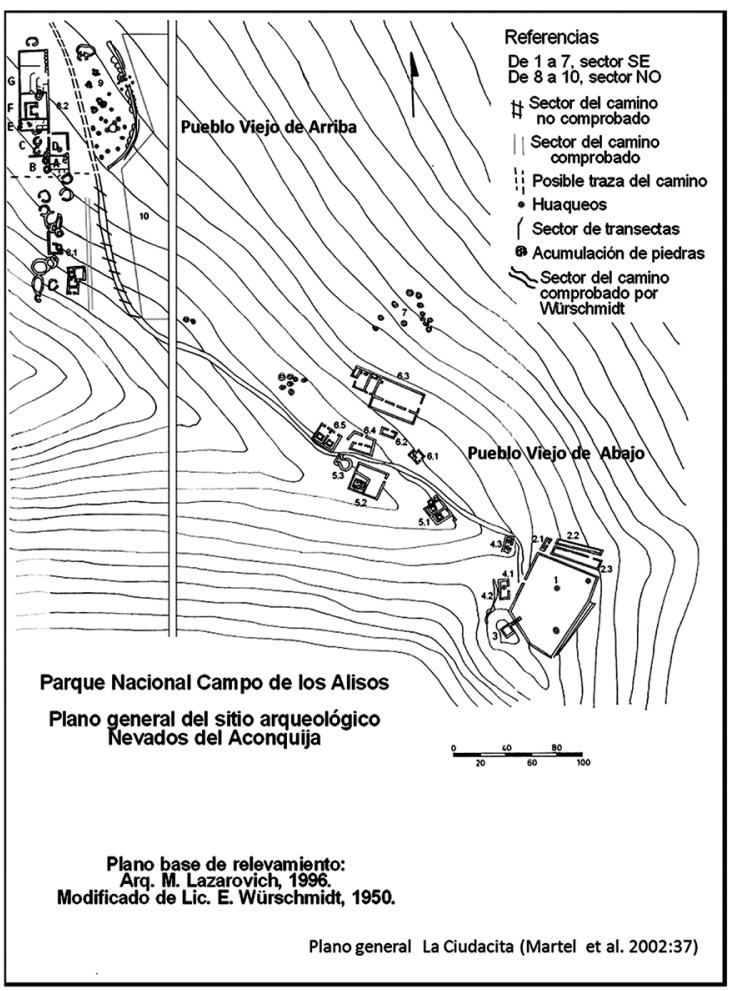

Figura 2. Plano general del sitio (Martel et al. 2002: 37). 
Dicha disposición —en dos sectores - llevaron a Hyslop y Schobinger (1991) a plantear una especie de dualidad simbólica, donde la presencia de estructuras típicamente incaicas, como kallankas, plazas intramuros, canchas y ushnu, representarían la dualidad de este "Nuevo Cuzco" entre el Hanan (arriba, lo inca) y el Hurin (abajo, lo local) (Ataliva et al. 2010: 176). El sitio recibe los nombres de "Pueblo Viejo del Aconquija", "La Ciudad Legendaria", "Ruinas de los Nevados", "Pucara de Las Pavas", "Nevados del Aconquija” o "La Ciudacita" (Ataliva et al. 2010: 161); es conocido desde 1875 gracias a un artículo de Federico Schickendantz, en la "Revista Mensual del Plata", donde se le nombraba como "una ciudad legendaria ubicada sobre las faldas orientales del nevado", y también por las referencias de Franz Mansfeld (1948) quien la visitó en 1937 (Beorchia 2001: 197; Ataliva et al. 2010: 164).

Dentro de una expedición científica, las ruinas fueron visitadas en el año 1948 por una comisión de la Universidad Nacional de Tucumán integrada por el profesor Guillermo Rohmeder y Osvaldo Paulotti. Éstos, en compañía de estudiantes de la misma facultad, realizaron el primer levantamiento topográfico y descripción del material en superficie ${ }^{12}$, asesorados por el arqueólogo Emilio Barbieri. La expedición siguió la ruta desde la vertiente tucumana, es decir, siguiendo Concepción, Alpachiri, Santa Rosa, La Mesada y Puesta Cascada, estableciendo

10 Cabe destacar que la diferencia altitudinal genera una amplitud térmica entre la llanura tucumana $\left(41^{\circ} \mathrm{C}\right)$ y la Ciudacita $\left(-6^{\circ} \mathrm{C}\right)$ en verano, lo que genera fuertes ráfagas de viento hasta los $110 \mathrm{~km} / \mathrm{h}$ (Bravo 1993: 7-8).

11 En palabras de Hyslop quien visitó el sitio en 1986 “...las ruinas incásicas más importantes, que él ha visto, desde el Cuzco al sur” (Bravo 1993: 7).

12 De la cerámica recolectada, corresponde ésta a un alto porcentaje Santamariano 35\%, Belén 32\% e Incaico 30\%. (Paulotti 1959: 126). Posteriormente en 2002, se identifican en superficie los tipos Belén Negro sobre Rojo, Santa María Bicolor, Famabalasto Negro sobre Rojo y un posible fragmento de Inca Imperial (Martel et al. 2002: 12).

13 La obtención de agua se realizaba a $300 \mathrm{~m}$ al noroeste de Pueblo Viejo de Arriba, junto a un ojo de agua canalizado que baja del cerro (Beorchia 2001: 212). Este sistema de acequias ya fue registrado por Mansfeld (1948), quien además plantea la posibilidad de la extracción de cuarzo en las cercanías de La Ciudacita (Martel et al. 2002:3-4).

14 Se define como el conjunto de sitios y/o hallazgos aislados, que se asumen interrelacionados y emplazados en zonas de cumbre, ladera o base de una montaña (Schobinger y Ceruti 2001: 530; Martel et al. 2002: 4). el campamento en el sector de Las Cuevas, desde donde subían diariamente al sitio en cuestión (Paulotti 1959, $1967)^{13}$. Gran parte de los planos con los que se cuenta corresponden a los esfuerzos del profesor Enrique Würschmidt (1952), quien entre 1948 y 1950 realizó el relevamiento de las estructuras arquitectónicas, dando incluso descripciones completas del clima y la edafología de la región. Se le reconoce como una "localidad arqueológica de altura"14 a partir del trabajo de Schobinger y Ceruti (2001), compuesto por diferentes estructuras arquitectónicas de variable complejidad y análogas a otras encontradas en el noroeste argentino (Martel et al. 2002: 3).

Entre las hipótesis funcionales del sitio destacan las de Mansfeld (1948), quien plantea la posibilidad de vetas de mineral en el área de La Ciudacita. La de Würschmidt (1952), quien propone que estas construcciones cumplieron funciones militares y religiosas, además de asiento para funcionarios del Tawantinsuyu (Ataliva et al. 2010: 164-165). Y la de Pioseek (1980), quien sugiere que "además de observatorio y centro astronómico y ceremonial, la Ciudacita era un puesto privilegiado de observación de movimientos que en la llanura tucumana pudieran hacer las tribus nómadas y depredadoras de origen amazónico [grupos lules]" (1980, en Bravo 1993: 13). Scattolin y Korstanje (1994) retoman la hipótesis de Hyslop y Schobinger, considerando a los Nevados de Aconquija como un establecimiento fronterizo, cuyo objetivo principal habría sido evitar que las poblaciones del oriente accedieran a la otra vertiente. En el mismo sentido, Williams (2000) incluye al sitio como un enclave administrativo o fortaleza (Martel et al. 2002: 5; Ataliva et al. 2010: 167). Nosotros concordamos con Hyslop y Schobinger, quienes señalan:

“... la ubicación estratégica del sitio, la presencia de varias kallankas y la necesidad que tuvo la administración incaica de proteger la frontera oriental, resultado de ello es la construcción del sitio aquí contemplado y del Pucara de Andalgalá, que se encuentra más al sur, en la provincia de Catamarca" (Hyslop y Schobinger 1991: 22).

En el primer grupo destaca la plaza principal o Calasasaya con seis muros pircados y una superficie de $300 \mathrm{~m}^{2}$ construidos, a $4384 \mathrm{msnm}$. En un plano elaborado por Würschmidt (1949-1950) y presentado por Bravo (1993: 9-10), las dimensiones de los muros son: BA (51,84 m), 
$\operatorname{AF}(60 \mathrm{~m}), \mathrm{FE}(24,08 \mathrm{~m}), \operatorname{ED}(53,60 \mathrm{~m}), \mathrm{DC}(23,58 \mathrm{~m})$ y $\mathrm{CB}(23,49 \mathrm{~m})$; vértices internos: $\mathrm{A}\left(86^{\circ} \mathrm{O} 3^{\prime}\right), \mathrm{B}\left(129^{\circ} 23^{\prime}\right)$, $\mathrm{C}\left(173^{\circ} 13^{\prime}\right), \mathrm{D}\left(72^{\circ} \mathrm{O} 0^{\prime}\right), \mathrm{E}\left(163^{\circ} 24^{\prime}\right)$ y F $\left(95^{\circ} 24^{\prime}\right)$; ancho de $0,65 \mathrm{~m}$ y altura de 1,10 a 1,25 $\mathrm{m}$ en promedio (Figura 3).

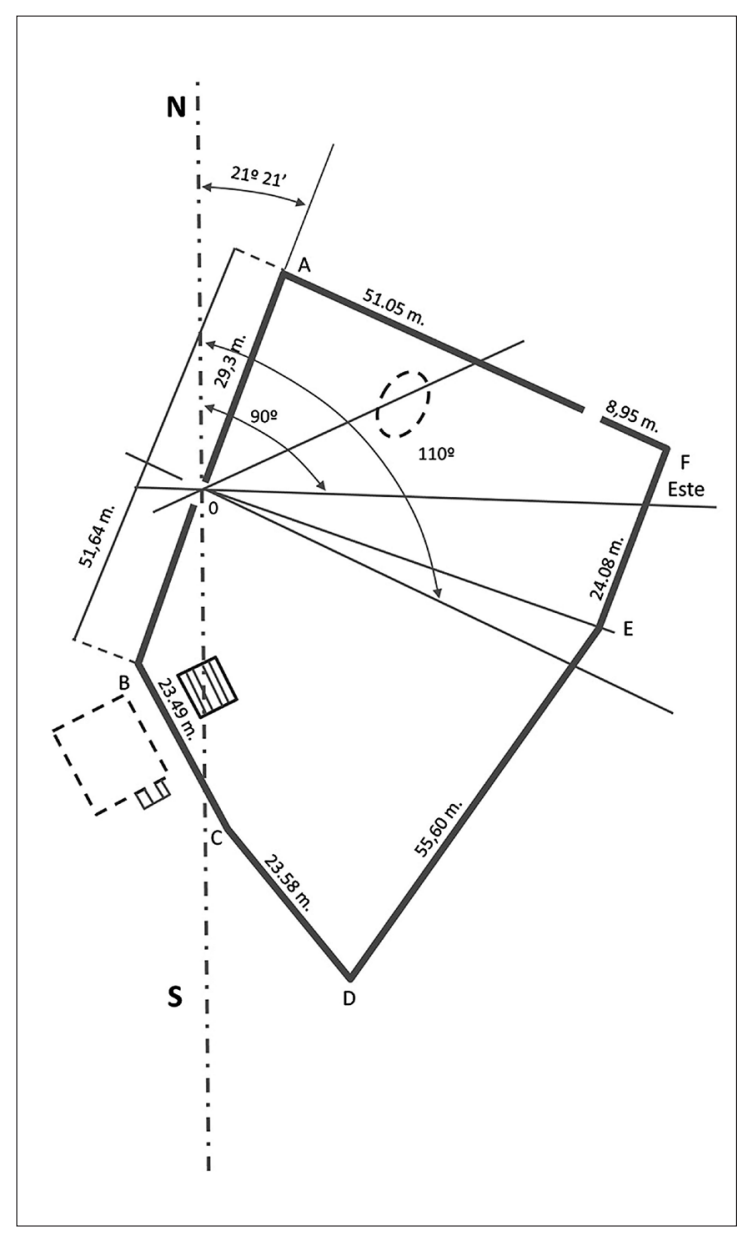

Figura 3. Plano Pueblo Viejo de Abajo (modificado de Bravo 1993: 10).

La técnica de construcción corresponde a piedras canteadas dispuestas en seco en capas horizontales, muros dobles, relleno con piedras pequeñas, rodados y tierra, y cimientos de $1 \mathrm{~m}$ de profundidad construidos a plomada. La plaza presenta dos puertas, una al oeste $(1,60 \mathrm{~m}$ altura, 1,10 $\mathrm{m}$ ancho muro y 1,16 $\mathrm{m}$ de ancho), provista de cuatro escalones, entre la cara interna y externa del muro. Cada escalón tiene 1,16 m de longitud, de 0,32 a 0,54 m de ancho y $0,10 \mathrm{~m}$ de alto. Formado por dos o tres lajas canteadas yuxtapuestas, que sirven para descender a la plaza. El otro acceso se encuentra en el muro norte y no difiere del anterior. Destaca en la parte sureste de la plaza un muro de contención de tres cuerpos de $51 \mathrm{~m}$ de largo, construido al borde del mismo precipicio. El primero de $1 \mathrm{~m}$ de altura y 1,7 $\mathrm{m}$ de ancho, el segundo de $0,95 \mathrm{~m}$ de alto y o, $8 \mathrm{~m}$ de ancho, y el tercero $1,4 \mathrm{~m}$ de altura y o, $8 \mathrm{~m}$ de ancho (Paulotti 1959: 127-129). Al centro de la plaza se advierte un doble círculo (elipse) de piedras irregulares y lajas, en cuyo centro se encontró enclavado un tosco monolito o menhir (hoy inexistente), cuyo círculo mayor es de 15,30 m (norte-sur) por 7,72 $\mathrm{m}$ (este-oeste), distante a 13,20 m del muro noroeste (BA). Este especie de menhir, gnomon o "pirámide rectangular trunca de cuatro lados" habría presentado una cara mayor de 0,26 $\mathrm{m}$ (ancho máximo) y 0,21 (ancho mínimo), y una cara menor de 0,16 m (ancho máximo) y o,12 (ancho mínimo) (Paulotti 1959: 129-130; Bravo 1993: 10).

En el extremo sudoeste (muro CB), se levanta un montículo semiartificial, cuya altura es de $5 \mathrm{~m}$ definido como un ushnu, en cuya parte más alta se encuentran restos de lo que pudo ser un pequeño edificio, hoy destruido (Figura 4). Las dimensiones del montículo en su cima son $15 \mathrm{~m}$ de frente por $10 \mathrm{~m}$ de ancho. Mientras que la base tiene $32,50 \mathrm{~m}$ de frente por $21 \mathrm{~m}$ de ancho. Sobre un terraplén se ubica una escalera de acceso al montículo. Esta se compone de 23 o 26 escalones (hoy muy destruidos), construida por piedras canteadas y yuxtapuestas ${ }^{15}$. Cada peldaño tiene $2 \mathrm{~m}$ de longitud por $0,40 \mathrm{~m}$ de ancho, con una altura de 0,20 a $0,25 \mathrm{~m}$ en promedio. Del edificio levantado sobre el montículo queda poca evidencia. Es cuadrado de $8 \mathrm{~m}$ de lado, ocupando la mitad de la terraza superior, desde donde se dominan los llanos de Tucumán en un arco de $100 \mathrm{~km}$ aproximadamente (Paulotti 1959: 130-131; Beorchia 2001: 209). Con respecto al ushnu, se sabe que:

"Esta estructura junto a la piedra que originariamente hubo en la plaza, conforma un típico ushnu incaico, y uno de estos complejos mejor conservados en el todo el Tawantinsuyu, no muy diferente del que puede verse en Wilcas Waman (Perú). El significado simbólico, ritual, astronómico y político del complejo ushnu es central

\footnotetext{
15 Hoy parte del muro y la escalinata han colapsado, haciendo difícil constatar el número de peldaños del ushnu (Martel et al. 2002: 14; Ataliva et al. 2010).
} 


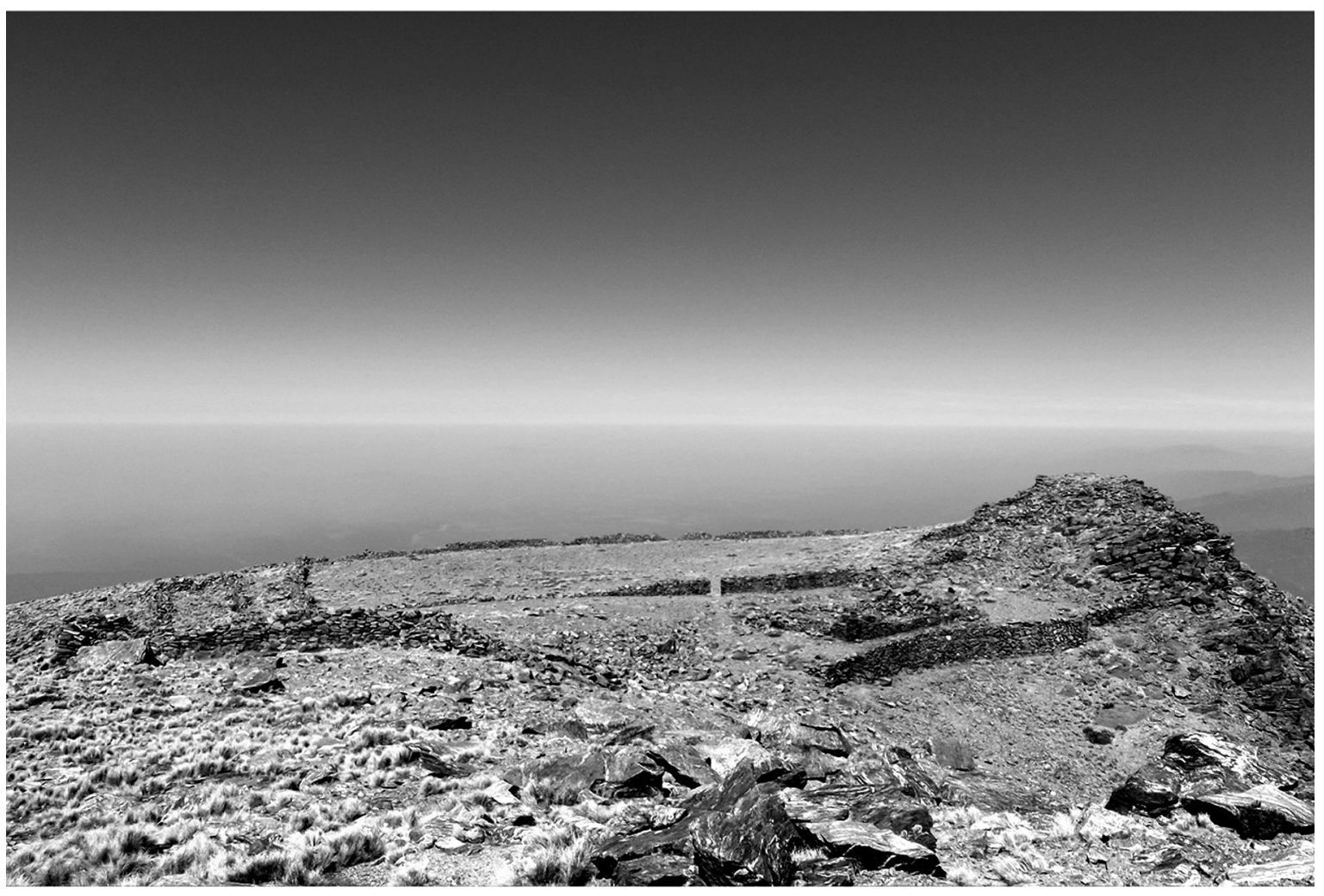

Figura 4. Vista general plaza y ushnu.

para el pensamiento incaico. Se trata de un concepto básico para entender el diseño de las grandes instalaciones incaica" (Hyslop Ms., citado en Beorchia 2001: 210).

Al respecto, podemos retomar la clasificación de González (1980), quien adscribe el sitio dentro de la categoría de "tambo principal o mixto" en su tipología de sitios incas del noroeste argentino, principalmente por la existencia del ushnu, descrito por Paulotti, que lo vincula directamente con las actividades religiosas y de culto llevadas a cabo por los incas dentro de su proceso de expansión del Tawantinsuyu en zonas meridionales (González 1980, en Martel et al. 2002: 4; Ataliva et al. 2010: 165).

El segundo grupo (Pueblo Viejo de Arriba), se encuentra a $600 \mathrm{~m}$ de Pueblo Viejo Abajo, sobre la ladera del cerro del Pino o cerro Bayo; comprende construcciones y recintos distribuidos en una superficie de $200 \mathrm{~m}$ por $70 \mathrm{~m}$, a una altitud de $4431 \mathrm{msnm}$, accediendo desde el llamado "camino del Inca". Aquí destaca la plaza o explanada (recinto $\mathrm{N}^{\circ} 11$ ) una superficie plana al este del camino men- cionado. Su forma es aproximadamente triangular, con el lado mayor junto al camino $(80 \mathrm{~m})$ y los lados menores, de $50 \mathrm{~m}$ cada uno, bordeando la barranca. Dentro de la misma se observan casi una veintena de apachetas (acumulación intencionales de piedras), alcanzando hasta los $3 \mathrm{~m}$ de diámetro cada una ${ }^{16}$. También se halló un gran canto clavado en el piso ( $1 \mathrm{~m}$ de largo x o,8 $\mathrm{m}$ de alto) tipo menhir, ubicado en el centro de la plaza y rodeado por un círculo de piedras planas ( $6 \mathrm{~m}$ diámetro y o,40 $\mathrm{m}$ elevado con respecto al piso), orientado este-oeste y posiblemente vinculado con la salida del sol en los equinoccios (Bravo 1993: 11; Paulotti 1967:364-369) 17 (Figura 5).

Hipótesis astronómicas surgen de la tarea del físico tucumano Orlando Bravo, quien destaca la posibilidad de tener un horizonte óptimo para la observación de los solsticios, con una amplitud angular de $52^{\circ}$ y una distancia de a lo menos $240 \mathrm{~km}$ al oriente. En 1984, con ayuda de una brújula magnética, verificó la orientación de la perpendicular del muro BA que coincide con los $113^{\circ}$ del vano de acceso a la plaza y la salida del sol para el solsti- 


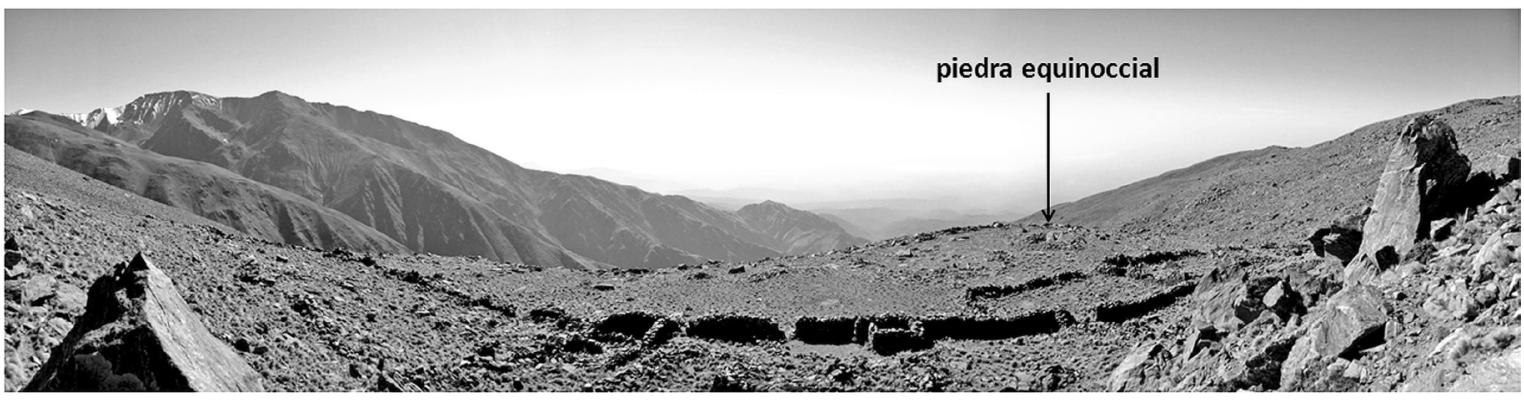

Figura 5. Vista general Pueblo Viejo de Arriba.

cio de diciembre. En 1985 se corrigieron las mediciones con ayuda del agrimensor Carlos Giovellina (Geodesia y Topografía, Facultad de Ciencias Exactas y Tecnología, UNT), obteniendo orientaciones con respecto al norte geográfico: muro $\mathrm{BA}\left(21^{\circ} 26^{\prime}\right)$, muro $\mathrm{AF}$ y eje puerta $\mathrm{BA}$ $\left(116^{\circ}\right)$, orientación plataforma (ushnu) a la esquina $\mathrm{F}$ $\left(64^{\circ}\right)^{18}$ (Bravo 1993: 10-11).

En la década de 1980 nuevas investigaciones reafirman la condición astronómica del sitio. Se realizan sendas expediciones en 1983 y 1986, la primera desde la ladera oriente, dirigida por Schobinger y Beorchia, y la segunda, por los mismos más Hyslop y Bárcena, pero esta vez desde la vertiente occidental de la Sierra (Ataliva et al. 2010: 166). El 22 de mayo de 1986, Beorchia y Hyslop observaron la puesta de sol desde el ushnu casi rozando la punta piramidal del cerro Las Cuevas (4900 msnm), donde en 1984 se descubrió una plaza amurallada con funciones posiblemente ceremoniales (Beorchia 2001: 197) ${ }^{19}$. Con este antecedente surge - entonces - la posibilidad que el sitio hubiera servido para conmemorar el solsticio de junio (inicio del invierno calendárico en el hemisferio sur). Los cálculos presentados plantean que para el año 1500 DC (horizonte $0^{\circ}$ ), las salidas y puestas del sol coincidían con los $63^{\circ} 03^{\prime}$ y $296^{\circ} 57^{\prime}$ de acimut, y que para una altura calculada de $6^{\circ}$, con respecto al plano del observador, estas cifras variarían a $58^{\circ} 49^{\prime}$ y $301^{\circ} 11^{\prime}$ de acimut (Beorchia 2001: 198-200 $)^{20}$.

Se plantea también la posibilidad de otro marcador al poniente, $54^{\circ}$ al sur del cerro Las Cuevas en un nevado (no identificado), donde se tienen noticias de "grandes montones de leña", para la puesta del sol en el solsticio de diciembre. Según cálculos realizados por Hyslop, este evento ocurriría $2^{\circ} 30^{\prime}$ al norte del nevado Hauthal (5340 msnm), coincidiendo con la falda oriental, del que se asume, es el cerro de Las Minas ( 5500 msnm) (Beorchia 2001: 205).

\section{La observación de ciclos lunares}

Desde tiempos remotos, la observación de los ciclos de la luna ha sido el fenómeno de la naturaleza más fácil de registrar, sirviendo como base para la estructura de distintos calendarios como el babilónico, el árabe, el hindú, el judío-hebreo y el chino (Schaefer 1992; King 1993; Montgomery 1999; Stern 2008; da Silva 2010). Estos sistemas calendáricos asumen períodos de tiempo (fijo) a partir de la combinación de los ciclos sinódico y sideral de la luna, constituyendo también base para los distintos sistemas de creencias a través de la creación de constelaciones, mitos y deidades lunares. Esta manera de conceptualizar el tiempo tuvo importancia dentro de los calendarios de santos en la Edad Media y actualmente

16 El Dr. Bravo relaciona estos "18 círculos" con la astronomía lunar y el metónico, igual a 19 años, que corresponde al momento cuando la luna se encuentra en su misma fase y lugar del cielo respecto al horizonte (Beorchia 2001: 206, 208). Sin embargo, también cabe la posibilidad que estas acumulaciones intencionales de piedra quedaran del proceso (inconcluso) de construcción del sitio, tras la caída del Tawantinsuyu en la primera mitad del siglo XVI (Ataliva et al. 2010: 182).

17 Este gnomon podría tratarse de una "wanka" o roca sagrada, en palabras de Schobinger (Beorchia 2001: 202).

18 SSSJ: salida sol solsticio de junio.

19 La observación de la puesta del sol para el solsticio de junio de 1996 confirmó que el evento astronómico ocurre $2^{\circ}$ al sur del cerro Las Cuevas. En dicha oportunidad, los expedicionarios tuvieron que moverse $50 \mathrm{~m}$ al norte del ushnu, ya fuera de la plaza, para que la puesta coincidiera con el marcador de horizonte (Beorchia 2001: 203, 205).

20 Estos cálculos difieren de los cálculos astronómicos realizados, pues no toman en cuenta el valor real del horizonte y la refracción atmosférica. 
incide en la fórmula para calcular la pascua cristiana en Occidente, coincidiendo con el domingo y primera luna llena después del equinoccio de marzo (Schaefer 1992).

Los ciclos lunares coinciden generalmente con los períodos de siembra y cosecha, maduración de los cultivos, crecidas de ríos y cuerpos de agua, así como con los ciclos fértiles de las hembras. En el mundo andino, la luna recibe el nombre de quilla en quechua o mara en aymara, y se le asocia también con la palabra "mes" (Ianiszewski 2010). Al respecto el Inca Garcilaso de la Vega, en sus "Comentarios Reales de los Incas", ofrece antecedentes sobre esta sincronía y la observación de los solsticios en el antiguo Perú:

"Porque contaron los meses por lunas, como luego diremos, y no por días y, aunque dieron a cada año doze lunas, como el año solar ecceda al año lunar como en onze días, no sabiendo ajustar el un año con el otro, tenían cuenta con el movimiento del Sol por los solsticios, para ajustar el año y contarlo, y no con las lunas. $Y$ desta manera dividían el un año del otro rigiéndose para sus sembrados por el año solar, y no por el lunar" (de la Vega 1945 [1609]: 111, en Bauer y Dearborn 1998: 57).

Su ciclo de fases, igual a 29,53 días, promediado en números enteros de 29 y 30 (Schaefer 1992; Stern 2008; Steele 2010), ha servido como un parámetro indicativo del tiempo social, al menos desde el Paleolítico Superior europeo en el sitio de Abri Blanchard, en Dordoña (Francia, con fechas cercanas al 29000 AC (Marshack 1972), y la Venus de Laussel (diosa de la fertilidad) con representaciones de 13 ciclos lunares dentro de un año, con fecha del 20000 AC (da Silva 2010, citando a Joseph 2010).

El ciclo sinódico de la luna (Figura 6) se inicia - arbitrariamente-después de 2,5 a 4,5 días de su invisibilidad (elongación $0^{\circ} / 360^{\circ}$ ), tras lo cual aparece como una delgada creciente por el oeste, cerca de donde se está ocultando el sol. Desde el punto de vista de la tierra, la luna avanza aproximadamente $13^{\circ} 10^{\prime} 35^{\prime \prime}$, lo que implica que la luna aparezca más tarde, día tras día, como una marcada creciente. La fase de primer cuarto (mitad del disco) se

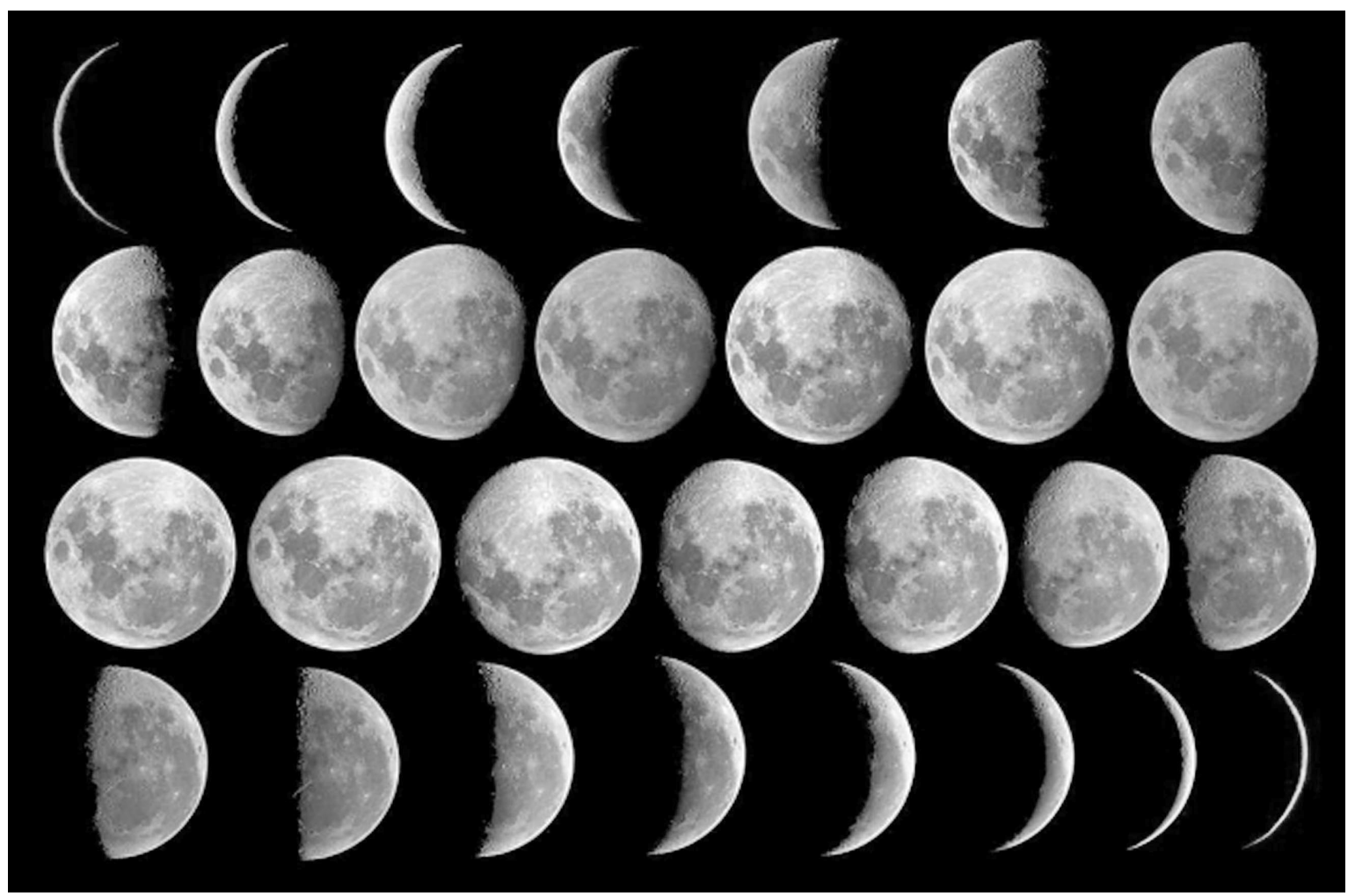

Figura 6. Ciclo sinódico de la luna (29,5 días). 
alcanza al séptimo día, cuando la luna alcanza una posición de $90^{\circ}$ con respecto al sol, consiguiendo su mayor altura en el meridiano celeste, al ocaso. A medida que la luna crece y alcanza su fase llena (días 14 - 15), ésta sale progresivamente más temprano y grande. En el día de la luna llena ésta coincidirá con la puesta de sol, pero opuesta en $180^{\circ}$. En la segunda mitad del ciclo la luna decrece, siendo visible sólo a altas horas de la noche y primeras de la mañana, y hacia el final del ciclo en pleno día, acercándose al punto donde sale el sol, hasta desaparecer por completo e iniciar de nuevo los días de invisibilidad (McCluskey 1986; Schaefer 1992: 33; Steele 2000: 9; Meeus 2002: 11-15; Aveni 2005: 98-100; Stern 2008).

Doce meses lunares sinódicos serán igual a 354 días, es decir, 11 días menos que la duración del año solar de 365 días. Este desfase permite que existan años de 12 y 13 lunaciones dentro de un año solar, fenómeno que no significó un problema para las antiguas culturas, siendo recurrente las cuentas lunares con 28 días, como entre los hopi y/o períodos de 29 y 30 días, p. e. el caso maya, cuya lógica parte de una luna nueva a otra luna nueva, o por sus fases llenas, considerando también meses intercalares cada 2,71 03 años, casi 37 lunaciones (Meeus 2002; Aveni 2005). El ciclo sideral corresponde al intervalo entre pasos sucesivos de la luna por una misma estrella o sector del cielo, con una duración moderna de 27,32 días, p. e. si vemos a la luna transitar por la constelación de Pléyades, estará en la misma posición 27,32 días después, pero en una fase y hora distinta. Este número fraccionario obliga al observador del cielo a tener en cuenta el $1 / 3$ de día (8 horas) de diferencia. Si la primera observación se realiza a media noche, la siguiente será cercana a las 8 am, 27 días y fracción después. Esta cuenta resulta en extremo difícil, pues el sol ya ha salido y han dejado de ser visibles las estrellas. La teoría indica que tal vez fue posible manejar un ciclo mayor, de tres meses siderales igual a 82 días $(3 \times 27,3=81,9)$, lo que permite ver a la luna (ligeramente desfasada) en una misma constelación y a la misma hora, a intervalos de tiempo sidéreo conocidos (Aveni 2005: 102).

Zuidema (2011) propone la existencia de un calendario lunar sideral para la organización sociopolítica del sistema de ceques del Cuzco. Su argumento se sustenta en la existencia de 328 lugares sagrados o huacas, igual al número de 12 meses lunares siderales $(12 \times 27 \cdot 3=327,6)$. El
328, numéricamente, se puede dividir en factores de 8 y 41 , que corresponden al número promedio de la semana andina y al número de ceques del Cuzco, respectivamente. El tiempo restante, igual a 37 días, necesario para alcanzar al año solar se completa con el tiempo ritual de invisibilidad de las Pléyades, entre el 3 de mayo y el 9 de junio. Período que se relacionaría con la cosecha y el almacenamiento del maíz en la latitud del Cuzco. Otros ciclos siderales de importancia en el mundo andino, pero no necesariamente lunares fueron los relacionados con la Cruz del Sur y el Cinturón de Orión (Zuidema 1982, 2011).

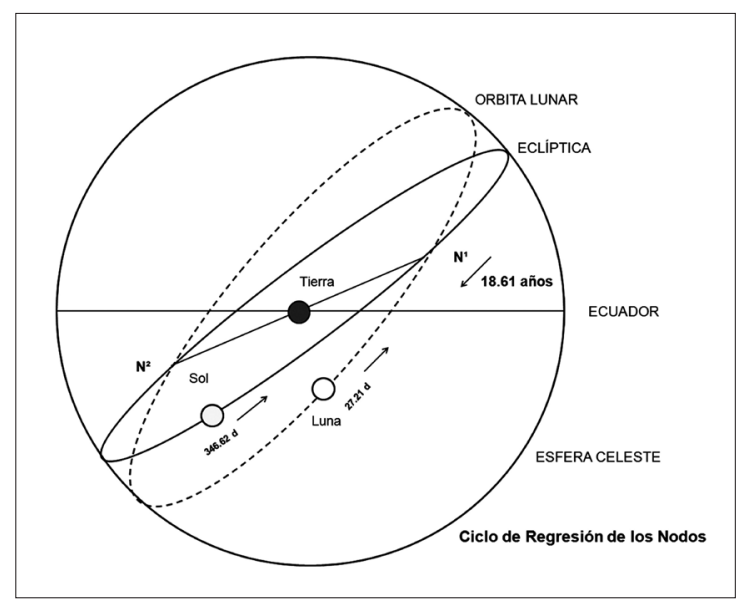

Figura 7. Regresión de los Nodos (18,61 años).

Un tercer movimiento de la luna tiene lugar por efecto de la inclinación de su órbita con respecto a la eclíptica, igual a $5^{\circ} 09^{\prime}$ en promedio ${ }^{21}$, con el cual puede alcanzar puntos sobre el horizonte un poco más al norte y al sur que el sol en su movimiento anual. A éste, se suma un leve bamboleo, con período de 173,31 días, cuya consecuencia es que la línea de intersección de ambos planos o "línea de los nodos" no esté fija, sino que tiene un movimiento, contrario a las manecillas del reloj, de 18,61 años (ciclo nodal), igual a 6797,15 días (Aveni 2005: 104) (Figura 7). Como consecuencia, dentro de un mes sinódico la luna ejecuta un movimiento sobre el horizonte, similar al del sol durante el año, pero con la diferencia que sus extremos no serán fijos, sino que varían no sólo cada mes, sino también dentro del ciclo nodal. De allí, que la luna no tendrá dos, sino cuatro detenciones (paradas) lunares

${ }^{21}$ Este valor puede variar entre $5^{\circ} 00^{\prime}$ y $5^{\circ} 19^{\prime}$. 


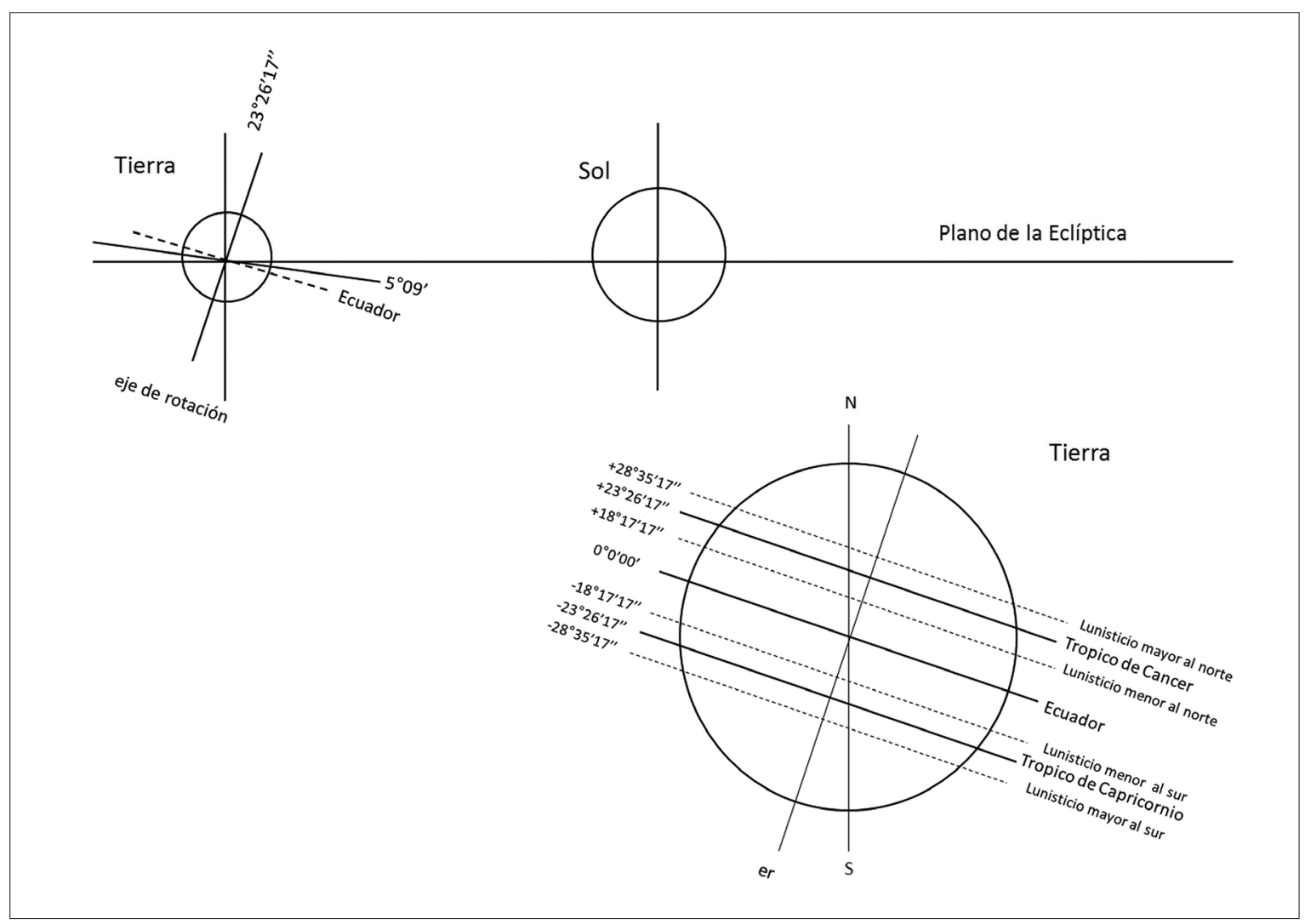

Figura 8. Plano de la eclíptica y trópicos lunares (modificado de Ianiszewski 2010).

o "lunisticios" dentro de cada ciclo nodal. Las declinaciones extremas serán igual al valor de la eclíptica $23^{\circ} 26^{\prime}$ $+5^{\circ} \circ 9^{\prime}$ al norte y al sur, es decir, $+28^{\circ} 35^{\prime}$ y $-28^{\circ} 35^{\prime}$ o lunisticios mayores. En otras palabras, la luna llena en el solsticio de invierno tomará el lugar del sol en el solsticio de verano y a la inversa, con una declinación de $\pm 23,5^{\circ}$. Los lunisticios menores ocurren cuando la luna alcanza valores de $-5^{\circ} 09^{\prime}$, al norte y al sur de la eclíptica, igual a $+18^{\circ} 17^{\prime}$ y $-18^{\circ} 17^{\prime}$, casi nueve años y medio más tarde (valor exacto: 9,305 años). La luna estará una media de unos siete años en cada par de lunisticios y unos dos o tres viajando entre ellos (Aveni 2005: 104-105; Belmonte 1999: 268-269; Lebeuf 2003: 150-151) (Figura 8).

Las paradas mayores y menores ocurren generalmente durante la fase de primer o tercer cuarto, que es cuando la luna alcanza su distancia mayor con respecto al sol en el movimiento de la eclíptica. Por un tema observacional resulta más fácil seguir a la luna en su fase llena, definida por Ianiszewski (2010) como "luna llena super tropical" en su momento más cercano al sur (Figura 9). Según este autor, esto sería consecuencia de que la luna en fase llena cruza el "internodo sur" o punto medio de la trayectoria lunar entre el nodo descendente y ascendente, junto con el punto más austral de la órbita lunar. Para que este fenómeno ocurra "deben coincidir los meses sinódicos, dracónicos y siderales en una fecha cercana al solsticio de junio [invierno]. A diferencia del eclipse, la luna no pasa por un nodo, sino que por el internodo sur, un lugar del cielo al sur del trópico de Capricornio, ubicado entre las constelaciones de Ofiuco y Sagitario, en la dec. 28,5 sur" (Ianiszewski 2010: 147).

Esta diferencia en grados - de la eclíptica - da origen a los eclipses, los que pueden ser solares o lunares. Los primeros, cuando la distancia al nodo es menor a 10, $3^{\circ}$, y los segundos, cuando la distancia al nodo es menor a $4,6^{\circ}$ (Green 1999). Diecinueve años de eclipses o 242 meses dracónicos (6585,35 días) están cercanos a 223 meses sinódicos (6585,32 días). Esto significa que la configu- 


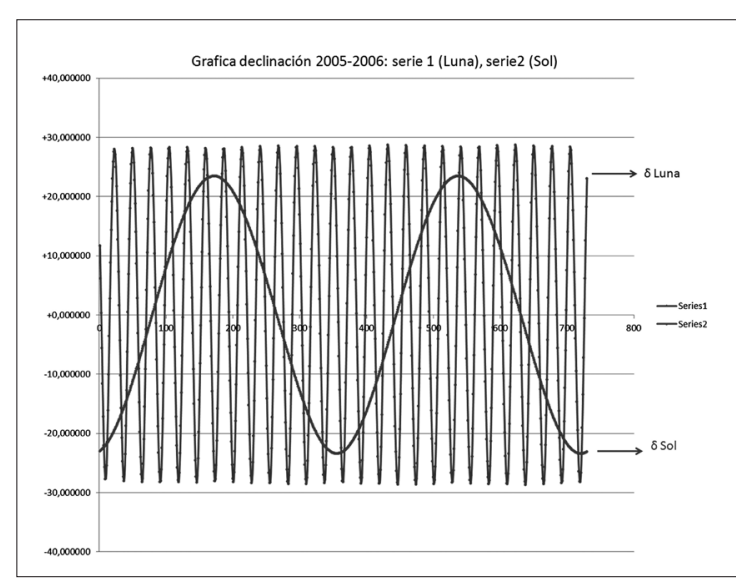

Figura 9. Gráfica de declinación lunisticio mayor (2005-2006) (Martz de la Vega et al. 2013).

ración Sol - Luna y los eclipses se repiten en el mismo orden, después de este período. Este ciclo fue conocido por los babilonios y por razones históricas se conoce como "Saros" con una duración de 18 años y 10,96 días. Tres ciclos Saros $(18,03$ x 3$)$ es igual a 54 años y 34 días, período necesario para que se repita el mismo eclipse en la misma parte de la Tierra (Exeligmos). Dentro de estos ciclos, destaca también el metónico que corresponde al ciclo de 19 años trópicos cuando la luna vuelve a la misma fase, en un mismo momento del año y más o menos con el mismo fondo estrellado ${ }^{22}$. Tiene una duración de 235 meses sinódicos (6939,68 días), 235+19 (254) meses siderales (6939,70 días) o 255 meses dracónicos (6939,11 días). Conocido desde tiempos babilónicos, siglo V AC como la imagen de la "serpiente de la luna llena" o grupo de 235 lunaciones, consecutivas, marcadas sobre un mapa de 31 estrellas conocidas y 35 ondas que cruzaba el zodiaco dos veces, coincidiendo con la eclíptica en momentos de peligro de eclipses lunares (Moesgaard 1980) (Tabla 1).

Según la tradición andina, sabemos que los incas creían en la existencia de dos bestias celestiales responsables de los eclipses, un león o una serpiente, lo cual supone el conocimiento del nodo. Aún más interesantes resultan las implicancias sociales del mismo, como atestigua el padre Bernabé Cobo:
"Acerca del eclipse tenían tantas boberías como del Sol: decían, cuando se eclipsaba, que un león o serpiente la embestía para despedazarla; $y$ por esto, cuando comenzaba á eclipsarse, daban grandes voces y gritos y azotaban los perros para que las diesen y aullasen. Poníanse los varones á punto de guerra, tañendo sus bocinas, tocando atambores, y dando grandes alaridos, tiraban flechas y varas hacia la Luna, y hacían grandes ademanes con lanzas, como si hubiesen de herir al león y sierpe; porque decían que desta manera los asombraban y ponían para que no despedazasen la luna" (Cobo 1892 [1653]: 328).

Polo de Ondegardo, refiere a este tipo de fenómeno y a otros observados en el cielo:

"Cuando se eclipsa el sol, ó la luna, ó parece algún cometa, ó resplandor en el ayre suelen gritar y llora, y hazer que otros griten y lloren, y que ladren los perros, ó aúllen y para esto los aporrean. Suelen cercar sus casas en procesión de noche con hazes de fuego y hazer otras ceremonias para que no les venga el mal que temen y que tienen por agüero malo" (Polo de Ondegardo 1916d: 198 [1567: Cap. 4], en Bauer y Dearborn 1998: 177).

Para los eclipses de luna se conocían dos palabras para definir el fenómeno:

"Lo que usaban antiguamente en los eclipses de la luna, que llaman Quillamhuañun, la luna se muere, o Quillatutayan, la luna se escurece, usan también ahora azotando los perros, tocando tambores y dando gritos por todo el pueblo para que resucite la luna" (Arriaga 1964[1621]: 218).

De acuerdo con Zuidema (1981, 1982, 1989), en el Cuzco habría tenido especial importancia la observación del sol en su puesta para los días del anti cenit (agosto 18 y abril 26). Estas fechas indican el momento de la siembra y la cosecha ritual, siendo marcadas por un conjunto de pilares en el cerro Picchu, que eran observados desde el ushnu de la plaza de Haucaypata. El mismo Zuidema (2011), a partir de la lectura de los cronistas Pizarro, Betanzos,

\begin{tabular}{|c|c|c|}
\hline Saros & Nodos & Metónico \\
\hline 18,03 años $^{23}$ & 18,61 años & 19 años \\
\hline 223 lunaciones & 230 lunaciones & 235 lunaciones \\
\hline
\end{tabular}

Tabla 1. Ciclos lunares. 
Anónimo y Molina, sugiere que el ushnu incluiría tres componentes: un pilón (fuente, agujero), una plataforma y posiblemente un pilar (gnomon). Para Cuzco, sugiere la existencia de dos ushnus: uno ubicado al norte de la ciudad en la plaza de Hanan-Cuzco cerca del edificio del Sunturhuasi, y el otro al sur en la plaza de Hurin-Cuzco, haciendo explícito los principios andinos de dualidad y cuadripartición (Zuidema 1980, 1989).

“... para tomar el punto del sol... tenían otro pilar en medio de la placa... en un paraje señalado a propósito, que le nombraban Osno y desde alli tomaban el punto del sol... y estando ajustando, hera tiempo general de sembrar... La luna de setiembre llamaban Cituaquilla. Este mes se juntaban en el Cuzco todos los yndios de la comarca, y juntos todos en la plaza principal, llamada Haocaypata, y alli hacían sus sacrificios al sol con muchas ceremonias en un pilar de piedra que tenían en medio de la placa, con su teatro llamado Osno y los hazían corderos y rropas de precio y otras muchas cosas y al pie del teatro vertían mucha chicha: dezian que la ofrecían al sol..." (Anónimo 1879 [1594]: 151ss., en Raffino et al. 1997: 28).

Ziólkowski y Lebeuf (1993) retoman la descripción del cronista Anónimo e intentan proponer un modelo de predicción de eclipses a partir de la posición de la luna cerca del nodo en sus paradas menores. Para ello, se fijan en dos aspectos centrales de la geometría de los pilares en Picchu:

- La diferencia ( $\boldsymbol{\delta}$ ) entre los pilares de agosto 18 y septiembre 2 es igual a $5^{\circ} 10^{\prime}$. Distancia cercana a la inclinación de la órbita con respecto a la eclíptica, igual a $5^{\circ} \circ 9^{\prime}$.

- La diferencia entre $18^{\circ} 10^{\prime}$ y $7^{\circ} 38^{\prime}$ es $10^{\circ} 32^{\prime}$, dos veces la distancia de inclinación de la órbita de la luna con respecto a la eclíptica.

Como consecuencia, si se observa la oscilación de la parada menor de la luna cerca de este pilar, se puede deducir

22 Fue descubierto por el astrónomo griego Metón en el siglo V AC., quien comprobó que las mismas fases lunares se repetían cada 19 años, siendo útil posteriormente para determinar la fecha de la pascua, en relación al puesto que ocupa cada año dentro de este ciclo (número Áureo). Presenta un error de 0,045 días después de 464 años, 0 0,0616 días cada 19 años (Meeus 2002:32-33).

23 Año trópico (valor moderno): 365,24219 días (Meeus y Savoie 1992).

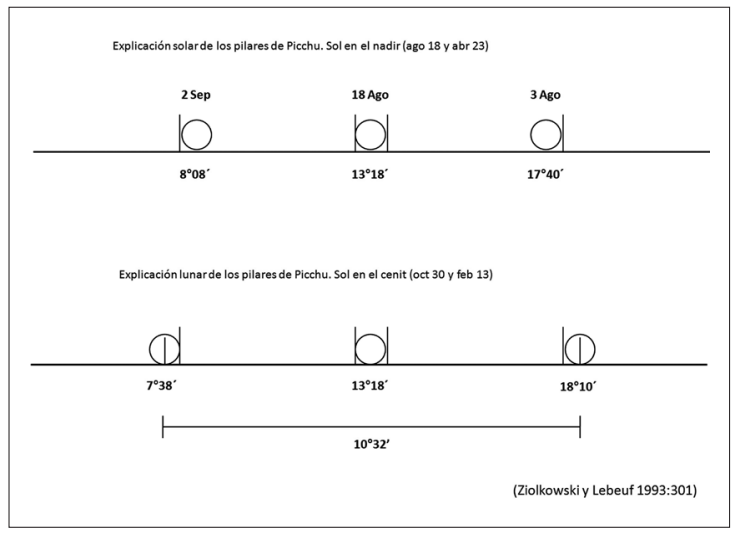

Figura 10. Orientación lunisolar de los pilares de Picchu (modificado de Ziólkowski y Lebeuf 1993: 301).

la variación de la inclinación lunar. Esto puede ocurrir para los días cercanos a febrero 13 y octubre 30 , cuando la luna cruza por los pilares centrales, donde la distancia es igual a $2^{\circ} 30^{\prime}$. Esta diferencia es similar a la distancia promedio entre una y otra puesta de la luna en esta parte de la eclíptica y al tamaño aproximado de la sombra de la tierra proyectada hacia la luna en los eclipses (Figura 10). Estas ideas se sustentan en un informe entregado por el padre Diego Rodríguez de Figueroa (1910 [1565]), misionero enviado por los españoles como embajador ante Titu Cusi Yupanqui, jefe del último bastión inca en Vilcabamba, en el cual al parecer se encuentran pistas de la supuesta predicción de un eclipse en tiempos incas. Según el clérigo, su llegada al campamento inca fue retrasada, llevándole fuera de él y haciéndolo esperar una noche a resguardo. Durante la noche el español habría escuchado ruidos y sonidos de flautas, además de gran alboroto:

"E luego otro dia que fueon honce de mayo, recibi otra carta del ynga... que yo fuese a otro pueblo mas adelante, que se llama Bambacona, para que mss presto no viwsemos, y que de ay s dos dhas" (Rodríguez Figueroa (1910 [1565]: 97, en Ziólkowski y Lebeuf 1993: 304-305).

Más adelante se señala:

"A catorze de mayo los yndios de Bambacona tenían tenían hecho una casa grande en un fuerte alto... E luego empeco á entrar la jente con el ynga en la placa" (Rodríguez de Figueroa 1910 [1565]) (Ziólkowski y Lebeuf 1993: 305). 
De acuerdo con Ziólkowski y Lebeuf (1993), durante la noche del 14 de mayo ocurrió un eclipse de luna visible en esta parte de los Andes ${ }^{24}$. Este vaticinio seguramente fue utilizado por el jefe inca para manejar el destino de la guerra contra los españoles, acordando una rendición pacífica. Solución diplomática sustentada en la creencia de que la luna eclipsada era un presagio de crisis y calamidad entre los incas. Estos antecedentes dan cuenta de una relación significativa entre la observación de un eclipse y determinadas prácticas sociales, como la guerra, pudiendo ser interpretado como un oráculo lunar relacionado con las nociones de la vida y la muerte en el mundo andino. Ideas que seguramente también se trasladaron a los eclipses de solares, donde era la propia autoridad del Inca la que se veía puesta en juego, al reconocerse el mismo como hijo de $\operatorname{Intt^{25}}$.

\section{El Inca y los espacios de frontera en Tucumán}

Como es sabido, la expansión del Tawantinsuyu estuvo determinada por la diversidad de las poblaciones y los territorios que entraron en contacto con los incas $y / o$ sus representantes. La continua expansión del Estado, con fechas iniciales ca. $1470 \mathrm{DC}$, para la parte sur o Collasuyu, creó numerosos y distintos escenarios en virtud de las diferencias sociales, políticas y económicas de las etnias conquistadas. Se establecieron así distintas y variadas formas de gobierno, intercambio y fronteras, que pueden ser explicadas desde las organizaciones locales y el rol del Estado, la sociopolítica, el ritual y calendario, entre otros, además de la necesidad continua por la obtención de mano de obra y recursos, en un territorio tan vasto como el comprendido entre el norte de Ecuador y el sur de Colombia, gran parte de Perú y Bolivia, el centro norte de Chile y el noroeste de Argentina (Dillehay y Netherly 1998: 3-10).

El Tucumán prehispánico se caracterizó por la ocupación multiétnica del espacio y funcionó como un conector entre los valles y las montañas situadas al oeste y la llanura oriental. En el piedemonte de la región conocida como el Tucumán se fusionaron tradiciones de tierras bajas

24 Eclipse parcial de Luna (Saros 104), magnitud 7,2: duración 82 min (http://eclipse.gsfc.nasa.gov/eclipse.html)

25 Para mayores referencias de eclipse solares y su significación en el mundo inca, ver Bauer y Dearborn (1998). y altas; según cronistas del siglo XVI y XVII, se encontraban aquí tres grupos principales: Lules, Tonocotés y Diaguitas (Noli 2012). En palabras de Lorandi (1998), esta región debió funcionar como un espacio de frontera, no en la perspectiva de su "marginalidad geográfica" con respecto al resto de los Andes, sino más bien al tipo de relaciones interétnicas al interior de la misma región. En el caso de Tucumán, estas relaciones son claras incluso antes de la llegada de los incas con los grupos juries, aconquijas y tafies, en la vertiente oriental de los nevados de Aconquija, quedando abiertas al contacto con poblaciones de las tierras bajas chaqueñas. En contraste con los grupos generalmente llamados "diaguita, de habla kakana" o "calchaquí", que habitaron las provincias de Salta, Catamarca, La Rioja y parte de la franja oeste de Tucumán, donde incluso existen evidencias de mitmaqkunas tucumanos en tiempos incaicos (Lorandi 1998: 197-199).

La incorporación de este territorio multiétnico al Tawantinsuyu, se debió, según algunos cronistas (Sarmiento 1960 [1572]; Pizarro 1986 [1571]; Betanzos 1996 [1551-1557]) a un interés minero. Esta afirmación puede sostenerse ya que tanto el noroeste argentino como el norte de Chile presentaron una importante tradición en la metalurgia del bronce desde antes del surgimiento del Estado inca, como también ocurrió una larga tradición de producción de lapidaria en minerales de cobre, turquesa, malaquita y atacamita (Williams et al. 2009). En un sentido amplio, y tomando como ejemplo otras áreas culturales, la región de Tucumán podría entenderse como un gran mosaico cartográfico. O conjunto de espacios, nichos y territorios, dispersos en el territorio, pero relacionados social y políticamente, gracias al control y demarcación del poder cuzqueño, ligado con aquellos aspectos y elementos sagrados de la topografía, como fueron los cerros huacas, que imprimían identidad y pertenencia territorial a las comunidades locales (sensu Sanhueza 2008: 59-60).

Para nuestro caso, cronistas indianos e historiadores consideran que la conquista por parte del Inca se habría realizado a partir del año $1470 \mathrm{DC}$, ocupando las actuales provincias de Jujuy hasta Mendoza. Raffino (2004) afirma que fue Tupac Yupanqui (1471-1493 DC), el conquistador de este sector del Tawantinsuyu. Los incas, para ello, se ubicaron en sitios estratégicos y construyeron 
fortalezas o pucaras, cuya función era frenar a los pueblos de la llanura chaqueña, chiriguanos y lules, que pugnaban en sus campañas depredadoras, desde el límite de las selvas tropicales (González 1980; Bazan 2004). Así, el Tawantinsuyu fue generando una infraestructura física para facilitar la administración estatal en los espacios conquistados, mientras que también recurrieron a la pacificación de los territorios intermedios para contener posibles grupos hostiles (Williams et al. 2009) ${ }^{26}$.

Los criterios económicos, y posiblemente también los ideológico-simbólicos, se impusieron en forma dialéctica con una redefinición del aparato de creencias surandino. En el proceso de ocupación de los nuevos espacios los planificadores cuzqueños mostraron gran flexibilidad para desarrollar sus objetivos particulares (González y Tarragó 2005). De esta forma, los líderes locales estaban dispuestos a prestar parte de su infraestructura y fuerza de trabajo comunitario para que el Estado pudiera organizar el nuevo espacio en construcción y practicar, así, una redistribución de bienes que se encontraba ligada al prestigio y experiencia de dichos líderes (Williams et al. 2009).

En el noroeste argentino se produjo además una marcación del paisaje simbólico, a través de adoratorios de alta montaña, mientras que en la parte suroriental del Collasuyu se distribuyeron asentamientos inca fortificados o situados en posiciones defensivas (Raffino 1981; Ceruti 1999). Se considera entonces que, durante el reinado de Huayna Capac (1493-1525 DC), la frontera suroriental del imperio se reforzó con el establecimiento de una línea de fortalezas para evitar las incursiones de los grupos chiriguanos. Así, los incas establecieron fronteras donde vivían grupos nómades o semisedentarios, y belicosos, que impedían la provisión de mano de obra para el Estado (Scattolin y Korstanje 1994). Dillehay y Netherly (1998) no descartan que los incas hayan mantenido relaciones comerciales o de otro tipo con los grupos que habitaron las selvas, aun cuando no hubiera un control efectivo de este territorio. Razón por la cual, gran parte de los asentamientos defensivos se ubicaron también en posiciones estratégicas para controlar el tráfico, a través de puntos clave naturales, especialmente pasos montañosos, p. e. Incallajta (Bolivia), cerro Grande de la Compañía (Chile) y pucara de Andalgalá (Argentina) (Williams et al. 2009) ${ }^{27}$.

Sabemos entonces que el Tawantinsuyu manipuló y cons- truyó el paisaje: articulando los procesos de dominación política y social a lo largo de su territorio, modificando elementos vitales del mismo y generando una transformación del orden social y cosmológico local, bajo nuevas pautas de concebir el espacio y la arquitectura en las provincias conquistadas (Leibowicz 2012). Lo cual significó, en resumidas cuentas, la incorporación no sólo de territorios y población efectiva, sino también de un conjunto de creencias y memorias colectivas ligadas a las nociones sagradas del territorio, a través de "nuevos Cuzcos" (Hyslop 1985), que conectaron simbólicamente todo un corpus de tradiciones vinculadas con el culto a la naturaleza, la observación del cielo y la sacralización de los espacios y lugares, p. e. algunos cerros, en esta parte meridional de los Andes.

\section{Análisis astronómico}

El análisis astronómico se realizó en base a instrumentos de observación (ushnu y piedra equinoccial), orientaciones arquitectónicas (cancha) y marcadores de horizonte descritos anteriormente por Paulotti (1967), Bravo (1993) y la expedición del CIADAM (Beorchia 2001), bajo la premisa que el sitio La Ciudacita, además de un lugar para observar al sol para solsticios y equinoccios, eventualmente pudiera haber servido para seguir la posición de la luna llena en su momento de parada mayor o menor, junto con el crossover del mes de septiembre.

Los resultados del cálculo de horizonte desde el ushnu de Pueblo Viejo de Abajo (Moyano 2013), son los expresados en la Tabla 2.

Aquí se constató la existencia de un marcador de horizonte al poniente, cercano a la puesta de sol en el solsticio de junio (PSSJ), eventualmente relacionado también con la puesta de la luna en su posición extrema norte (PLEN), en cerro Las Cuevas (4900 msnm) (v: 16 $06{ }^{\prime} 58.36^{\prime \prime}$, h:

26 Estas ideas concuerdan con las nociones de la conquista inca en territorios selváticos, donde más bien se dedicaron al intercambio de productos, p. e. animales, plantas, frutos y/o plumas, entre otros, más que a un control efectivo, quizás debido al nivel de complejidad social de las poblaciones locales (Dillehay y Netherly 1998: 10).

27 Este último llamado también "Fuerte de Andalgala o Pucara de Aconquija”, al sur de La Ciudacita, destaca por la presencia del Rectángulo Perimetral Compuesto (R.P.C.) y la red vial inca, inscrito en un enclave de frontera a los pies de los Nevados de Aconquija (Raffino 1981: 73-136). 
$\left.311^{\circ} 25^{\prime} 24^{\prime \prime}\right)$. Aquí se conocen evidencias de ruinas de tipo ceremonial (plaza) desde la década de 1980, reafirmando la existencia de una posible huaca en el lugar con su respectivo ceque (línea de visión) (Beorchia 2001) (Figura 11).

Esta orientación $\left(\delta+25^{\circ} 22^{\prime} 37.55^{\prime \prime}\right)$ pudo relacionarse con al menos tres eclipses ocurridos entre 1424 y 1479 $\left(\right.$ Saros 106) ${ }^{28}$ (Figura 12), que coincidieron la salida de la luna por la esquina $\mathrm{F}$ de la cancha y una puesta -de la luna- por la falda sur del cerro Las Cuevas, muy cerca de donde se debería observar la puesta del sol para el solsticio de junio (PSSJ). Esta especie de simetría pudo referir a la necesidad de los incas y/o los constructores del sitio, de resaltar la condición sagrada del solsticio, orientando la esquina $\mathrm{F}$ (vista desde el ushnu) a la salida del sol, y de forma complementaria, buscando la coincidencia de la luna al poniente (en momentos del eclipse) sobre las faldas del cerro Las Cuevas. La misma esquina F marca la dirección de la salida del sol en el solsticio de junio (acimut $64^{\circ}$ ), que visto desde la posición del ushnu ocurre al sur del cerro Nuñorco (Grande y Chico), ubicado en la zona de Tafí del Valle.

El análisis de los planos del sector de la cancha (Pueblo Viejo de Abajo) y las medidas tomadas en terreno, constataron la orientación del vano del muro BA, hacia la salida del sol en el solsticio de diciembre (SSSD) (Bravo 1993; Beorchia 2001). Esta orientación seguramente relacionó la fiesta del solsticio de verano, el Capaq Raymi, con la realización de ceremonias públicas, gracias a la direccionalidad que habría tenido el ingreso, por parte de los asistentes, a la gran plaza o cancha justo al amanecer.

Las observaciones astronómicas con teodolito realizadas desde el ushnu confirman, además, la existencia de marcadores al poniente, en los cerros:

Chimberi para la puesta de la luna en su posición extrema sur (PLES) (v: $08^{\circ} 14^{\prime} 6.8^{\prime \prime}$, h: $\left.241^{\circ} 29^{\prime} 24^{\prime \prime}\right)$, es decir, al lunisticio mayor.

Tipillas para los días 12 de abril y or de septiembre (v: $09^{\circ} 37^{\prime} 38.25^{\prime \prime}$, h: $\left.284^{\circ} 55^{\prime} 24^{\prime \prime}\right)$. Orientación posiblemente relacionada con los eclipses del grupo de Saros 115 (8 y 18 de febrero de 1487 y 1505), momento en que la luna llena se ubicó eclipsada o al finalizar el eclipse muy cerca de la cumbre del Tipillas ${ }^{29}$ (Figura 12).

28 http://eclipse.gsfc.nasa.gov/LEcat5/LE1401-150o.html (consultado el 28 de mayo de 2012).

29 http://eclipse.gsfc.nasa.gov/LEcat5/LE1401-1500.html (consultado el 19 de noviembre de 2012).

\begin{tabular}{|c|c|c|c|c|c|c|c|}
\hline Punto & Nombre & Vertical & Horizontal & Declinación $(\delta)$ & Fecha & Observaciones & $(\delta)$ Paralaje lunar \\
\hline 1 & Las Cuevas & $16^{\circ} 06^{\prime} 58.36^{\prime \prime}$ & $311^{\circ} 25^{\prime} 24^{\prime \prime}$ & - & - & ca. PLEN & $+25^{\circ} 22^{\prime} 37.55^{\prime \prime}$ \\
\hline 2 & - & $04^{\circ} 33^{\prime} 35.07^{\prime \prime}$ & $337^{\circ} 20^{\prime} 24^{\prime \prime}$ & $+51^{\circ} 26^{\prime} 32.99^{\prime \prime}$ & - & - & - \\
\hline 3 & - & $04^{\circ} 51^{\prime} 53.88^{\prime \prime}$ & $00^{\circ} 58^{\prime} 24^{\prime \prime}$ & $+57^{\circ} 56^{\prime} 23.73^{\prime \prime}$ & - & ca. norte & - \\
\hline 4 & - & $05^{\circ} 02^{\prime} 03.97^{\prime \prime}$ & $25^{\circ} 30^{\prime} 24^{\prime \prime}$ & $+49^{\circ} 26^{\prime} 09.58^{\prime \prime}$ & - & - & - \\
\hline 5 & - & $(-) \mathrm{O}^{\circ} 30^{\prime} 49.39^{\prime \prime}$ & $48^{\circ} 40^{\prime} 24^{\prime \prime}$ & $+37^{\circ} 21^{\prime} 54.92^{\prime \prime}$ & - & - & - \\
\hline 6 & - & $(-) 02^{\circ} 24^{\prime} 24.99^{\prime \prime}$ & $52^{\circ} 47^{\prime} 24^{\prime \prime}$ & $+33^{\circ} 49^{\prime} 31.2^{\prime \prime}$ & - & - & - \\
\hline 7 & - & $(-) 00^{\circ} 49^{\prime} 55.34^{\prime \prime}$ & $181^{\circ} 25^{\prime} 24^{\prime \prime}$ & $-61^{\circ} 57^{\prime} 10.72^{\prime \prime}$ & - & ca. sur & - \\
\hline 8 & - & $09^{\circ} 08^{\prime} 5.64^{\prime \prime}$ & $234^{\circ} 32^{\prime} 24^{\prime \prime}$ & $-35^{\circ} 35^{\prime} 39.97^{\prime \prime}$ & - & - & - \\
\hline 9 & - & $08^{\circ} 14^{\prime} 6.8^{\prime \prime}$ & $241^{\circ} 29^{\prime} 24^{\prime \prime}$ & $-29^{\circ} 03^{\prime} 18.95^{\prime \prime}$ & - & ca. PLES & $-29^{\circ} 28^{\prime} 36.93^{\prime \prime}$ \\
\hline 10 & - & $07^{\circ} 27^{\prime} 44.85^{\prime \prime}$ & $243^{\circ} 06^{\prime} 24^{\prime \prime}$ & $-27^{\circ} 16^{\prime} 38.17^{\prime \prime}$ & - & ca. cenit lunar & $-27^{\circ} 42^{\prime} 07.91^{\prime \prime}$ \\
\hline 11 & - & $07^{\circ} 50^{\prime} 56.23^{\prime \prime}$ & $245^{\circ} 12^{\prime} 24^{\prime \prime}$ & $-25^{\circ} 35^{\prime} 22.55^{\prime \prime}$ & - & - & - \\
\hline 12 & - & $07^{\circ} 28^{\prime} 44.85^{\prime \prime}$ & $247^{\circ} 45^{\prime} 24^{\prime \prime}$ & $-23^{\circ} 09^{\prime} 43.37^{\prime \prime}$ & $14 \mathrm{dic} / 31 \mathrm{dic}$ & - & - \\
\hline 13 & C. Punta & $10^{\circ} 39^{\prime} 57.24^{\prime \prime}$ & $255^{\circ} 48^{\prime} 24^{\prime \prime}$ & $-17^{\circ} 23^{\prime} 28.05^{\prime \prime}$ & olfeb/11nov & - & - \\
\hline 14 & - & $09^{\circ} 19^{\prime} 32.31^{\prime \prime}$ & $259^{\circ} 29^{\prime} 24^{\prime \prime}$ & $-13^{\circ} 32^{\prime} 27.53^{\prime \prime}$ & $13 \mathrm{feb} / 3 \mathrm{ooct}$ & - & - \\
\hline 15 & - & $09^{\circ} 43^{\prime} 25.2^{\prime \prime}$ & $260^{\circ} 35^{\prime} 24^{\prime \prime}$ & $-12^{\circ} 44^{\prime} 19.09^{\prime \prime}$ & $15 \mathrm{feb} / 27 \mathrm{oct}$ & - & - \\
\hline 16 & - & $07^{\circ} 13^{\prime} 37.83^{\prime \prime}$ & $267^{\circ} 02^{\prime} 24^{\prime \prime}$ & $-05^{\circ} 54^{\prime} 50.69^{\prime \prime}$ & $06 \mathrm{mar} / 090 \mathrm{ct}$ & - & - \\
\hline 17 & - & $08^{\circ} 38^{\prime} 16.3^{\prime \prime}$ & $277^{\circ} 00^{\prime} 24^{\prime \prime}$ & $+02^{\circ} 12^{\prime} 59.28^{\prime \prime}$ & $27 \mathrm{mar} / 18 \mathrm{sep}$ & - & - \\
\hline 18 & - & $08^{\circ} 54^{\prime} 23.41^{\prime \prime}$ & $27^{\circ} 742^{\prime} 24^{\prime \prime}$ & $+02^{\circ} 42^{\prime} 4.86^{\prime \prime}$ & $28 \mathrm{mar} / 16 \mathrm{sep}$ & - & - \\
\hline 19 & - & $08^{\circ} 56^{\prime} 23.65^{\prime \prime}$ & $278^{\circ} 33^{\prime} 24^{\prime \prime}$ & $+03^{\circ} 25^{\prime} 33.94^{\prime \prime}$ & $30 \mathrm{mar} / 15 \mathrm{sep}$ & - & - \\
\hline 20 & C. Tipillas & $09^{\circ} 37^{\prime} 38.25^{\prime \prime}$ & $284^{\circ} 55^{\prime} 24^{\prime \prime}$ & $+08^{\circ} 35^{\prime} 45.12^{\prime \prime}$ & 12abr/olsep & Huaca & - \\
\hline 21 & - & $07^{\circ} 15^{\prime} 38.61^{\prime \prime}$ & $296^{\circ} 40^{\prime} 24^{\prime \prime}$ & $+19^{\circ} 46^{\prime} 44.61^{\prime \prime}$ & $20 \mathrm{may} / 25 \mathrm{jul}$ & - & - \\
\hline
\end{tabular}

Tabla 2. Cálculo La Ciudacita (ushnu). fecha: $21 / 09 / 11 \delta$ (lat): $27^{\circ} 10^{\prime} 52.4^{\prime \prime} \mathrm{S}=-27.181222 \lambda$ (lon): 66 $00^{\circ} 24.5^{\prime \prime} \mathrm{W}=$ -66.006805 alt.: $4384 \mathrm{msnm}$ (GMT-3). 


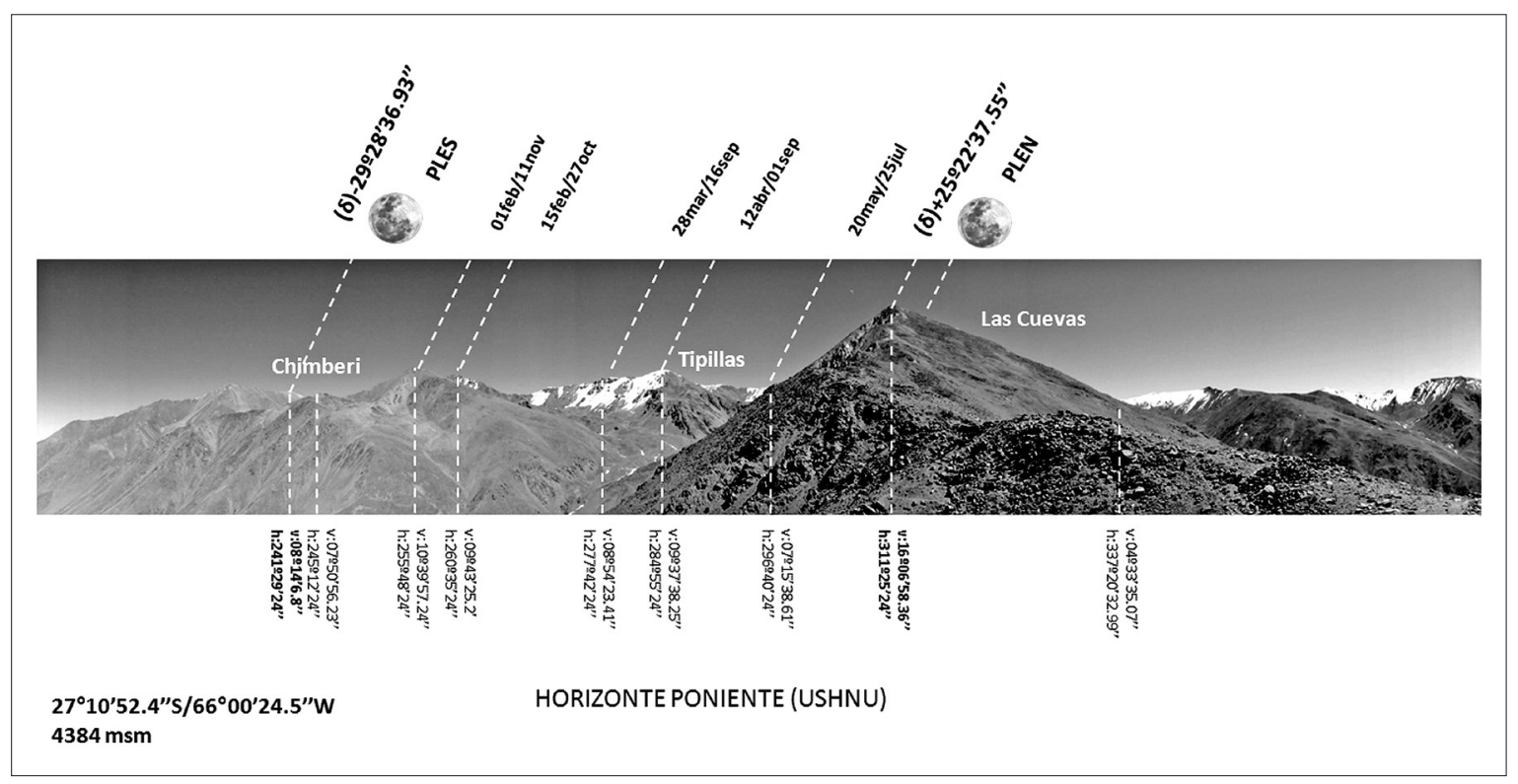

Figura 11. Horizonte poniente ushnu (Moyano 2013).

En segundo lugar, se constataron orientaciones solares desde una piedra - tipo gnomon- descrita por Bravo (1993) y Paulotti (1967) como piedra equinoccial. Los resultados son los expresados en la Tabla 3 (Moyano 2013).

En el lugar se obtuvo una medición que corresponde al eje mayor $\mathrm{AB}$ con un acimut de $80^{\circ} 31^{\prime} \mathrm{O} 0^{\prime \prime}$ en la horizontal y una declinación de (+) $08^{\circ} 15^{\prime} 44.26^{\prime \prime}$, marcador de las fechas de 11 de abril y o2 de septiembre, es decir, 20 días después y antes de los equinoccios de otoño y primavera (P-SSEQ), respectivamente (Figura 13). Este fenómeno pudo, eventualmente, ser percibido por los antiguos observadores del cielo en La Ciudacita, debido a la posibilidad que éste ofrece para determinar la intercalación de una 13a luna dentro de un año solar. Al poniente, el gnomon ofrece la posibilidad de marcar la posición del sol sobre el cerro Las Cuevas (v: $26^{\circ} 03^{\prime} 49 \cdot 19^{\prime \prime}$, h: $\left.296^{\circ} 12^{\prime} 00^{\prime \prime}\right)$, con una declinación de $(+) \circ 8^{\circ} 45^{\prime} 11.81^{\prime \prime}$, es decir, para los días 13 de abril y 31 de agosto (P-PSEQ), astronómicamente coherente con la observación del sol al oriente. Este tipo de orientación se explica en parte por el fenómeno del crossover entre el Sol y la Luna (fase llena) en momentos cercanos a los equinoccios. Definido como el momento cuando ambos astros, distantes en $180^{\circ}$ de diferencia pasan a ocupar el lugar opuesto en el cielo, de norte a sur o de sur a norte, al momento de la salida o puesta (Silva y Pimienta 2012: 197-203) $)^{30}$.

Curioso resulta entonces pensar que los antiguos observadores del cielo en La Ciudacita, no sólo miraron al Sol y la Luna, sino también contabilizaron sus movimientos para el conocimiento exacto del mes lunar y el momento de eclipses. Otro dato, no menos importante, son

30 En este caso, marcando posiblemente la posición de la luna llena del equinoccio de primavera (septiembre).

\begin{tabular}{|c|c|c|c|c|c|c|c|}
\hline Punto & Nombre & Vertical & Horizontal & Declinación $(\delta)$ & Fecha & Observaciones & $\begin{array}{l}\text { (ठ) Paralaje } \\
\text { lunar }\end{array}$ \\
\hline 1 & P. Equinoccial & $-00^{\circ} 21^{\prime} 34.86^{\prime \prime}$ & $80^{\circ} 31^{\prime} 00^{\prime \prime}$ & $+08^{\circ} 15^{\prime} 44.26^{\prime \prime}$ & $11 \mathrm{abr} / \mathrm{ozsep}$ & P-SSEQ & - \\
\hline 2 & C. Las Cuevas & $26^{\circ} 03^{\prime} 49.19^{\prime \prime}$ & $296^{\circ} 12^{\prime} 00^{\prime \prime}$ & $+08^{\circ} 45^{\prime} 11.81^{\prime \prime}$ & $13 \mathrm{abr} / 31 \mathrm{ago}$ & P-PSEQ & - \\
\hline 3 & - & $05^{\circ} 01^{\prime} 5.95^{\prime \prime}$ & $33^{\circ} 13^{\prime} 00^{\prime \prime}$ & $+44^{\circ} 32^{\prime} 41.06^{\prime \prime}$ & - & - & - \\
\hline
\end{tabular}

Tabla 3. Cálculo La Ciudacita (piedra equinoccial). fecha: $22 / 09 / 11 \delta$ (lat): $27^{\circ} 10^{\prime} 23.9^{\prime \prime} \mathrm{S}=-27.173305 \lambda$ (lon): 66 00 '44.1” W = -66.01225 alt.: $4431 \mathrm{msnm}(\mathrm{GMT}-3)$. 


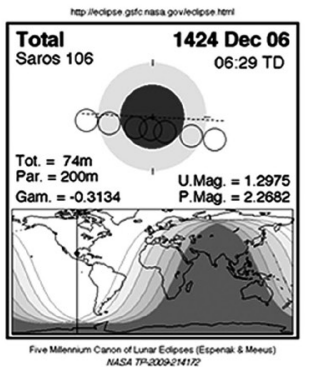

Saros 115
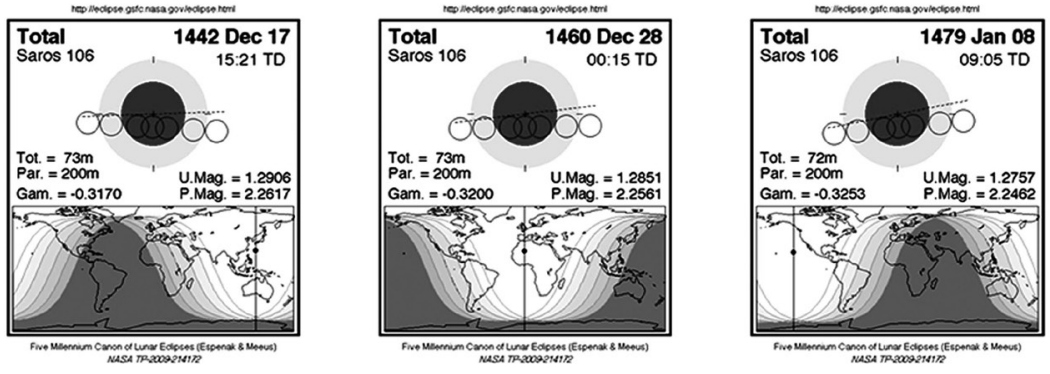

Marcador cerro Tipillas $\left(\delta+08^{\circ} 35^{\prime} 45.12^{\prime}\right)$

\section{Luna $\left(\delta+11^{\circ} 05^{\prime} 23.33^{\prime \prime}\right)$}

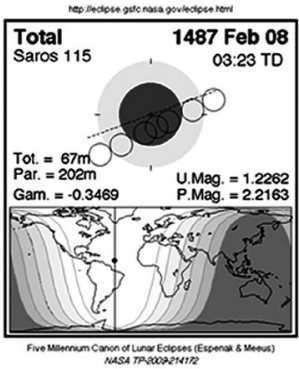

Luna $\left(\delta+06^{\circ} 57^{\prime} 47.25^{\prime \prime}\right)$
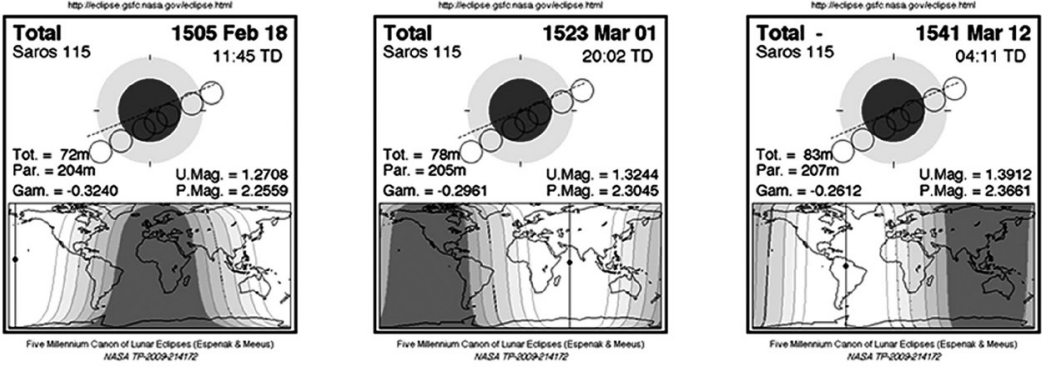

Figura 12. Eclipses lunares visibles Saros 106 y 115 (NASA).

los fenómenos visuales en estas altitudes, destacando la refracción atmosférica para la salida del sol donde la diferencia relativa, entre el punto de observación (a más de $4000 \mathrm{msnm}$ ) y la existencia de un horizonte lejano y bajo como es la zona del llano tucumano, permiten tener un horizonte de observación a poco más de $200 \mathrm{~km}$ de distancia ${ }^{31}$ (Figura 14). Esto genera el achatamiento del disco solar, a manera de cuerpos escalonados, instantes previos a la salida del astro por el horizonte, dentro de un momento simbólicamente importante, posiblemente en

31 http://marcosmolina.wordpress.com/tag/refraccion-atmosferica/ (consultado el 15 de mayo de 2012).

32 En la zona de Tafí del Valle se ha asociado este tipo de rasgos a la presencia de un culto generalizado a la Pachamama y la deidad solar, particularmente en montículos, campos de cultivo y sistemas de irrigación en campos agrícolas, al menos desde el 1000 DC (Sampietro et al. 2008). una hierofanía o manifestación de lo sagrado (Bustamante 2006, 2007, 2008).

Un dato a tomar en consideración es la supuesta existencia de un menhir (monolito vertical a manera de gnomon $)^{32}$ ubicado en el centro de la plaza principal en Pueblo Viejo de Abajo (Paulotti 1959: 127); éste, al igual que la piedra equinoccial, pudo haber cumplido funciones astronómicas relacionadas con el cenit, en particular para la observación de lunas llenas super tropicales, en latitudes cercanas a la parada mayor de la luna al sur.

\section{* Comentarios finales}

El sitio arqueológico La Ciudacita presenta características excepcionales para la observación del cielo, entre las que destacan: su altitud relativa con respecto al nivel del mar, la ausencia de nubes en determinadas épocas del 


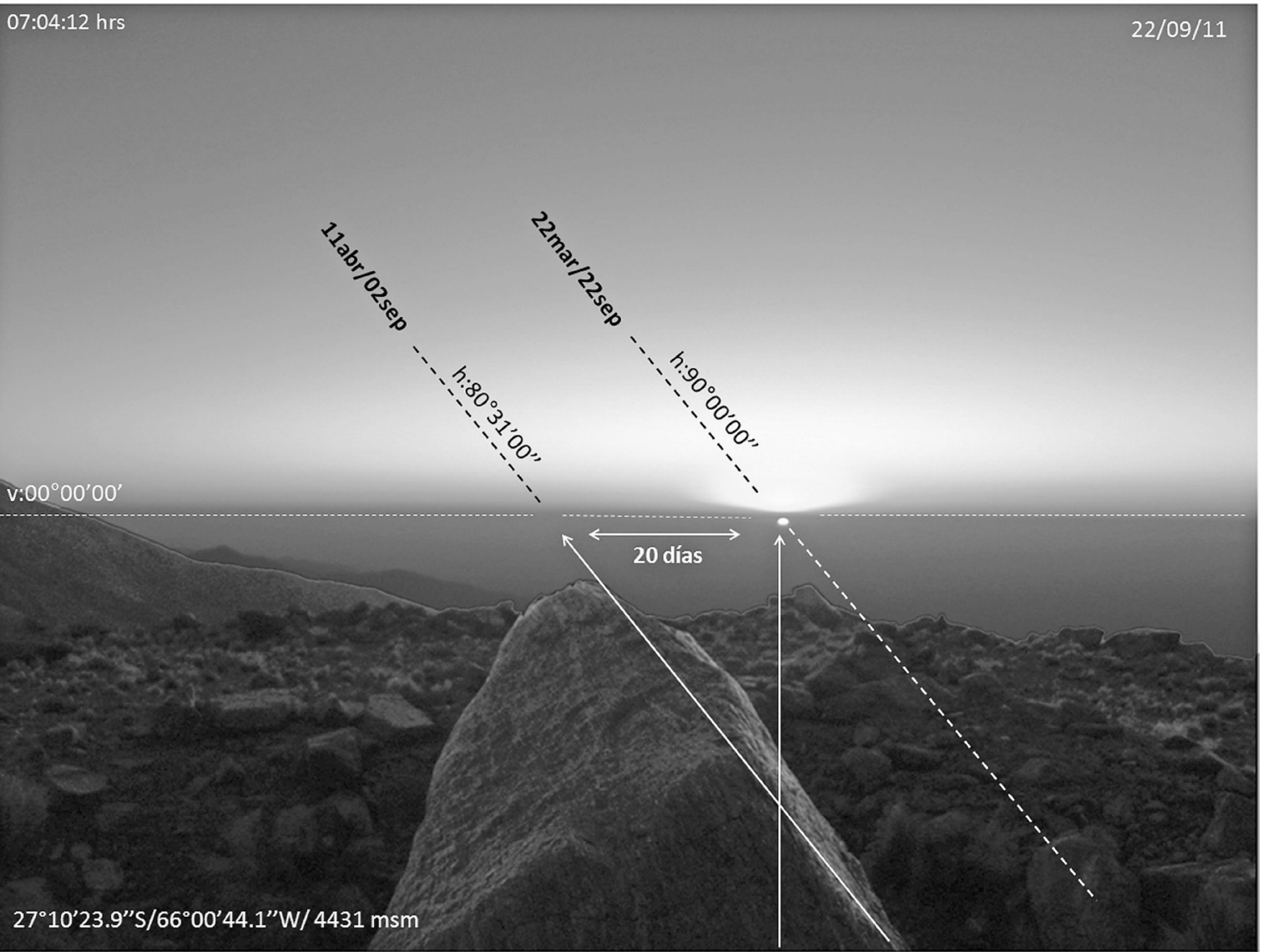

Figura 13. Horizonte oriente piedra equinoccial (Moyano 2013).

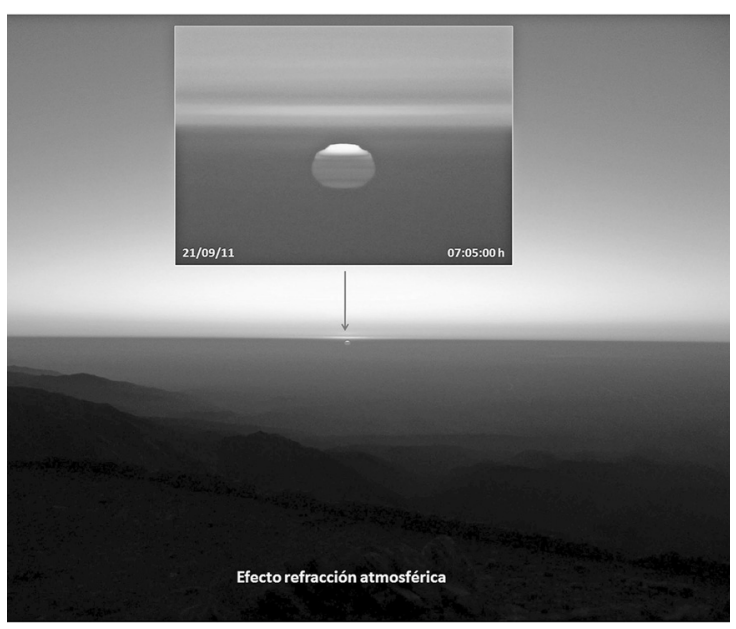

Figura 14. Efecto de la refracción atmosférica. año, la existencia de marcadores de horizonte (necesarios para la construcción y manejo de un sistema de calendario) y su posición geográfica latitudinal al sur del trópico de Capricornio (fenómeno del lunisticio en el cenit). Constituye en sí un tinkuy o punto de encuentro, debido a la confluencia de los ríos Jaya al este y Las Pavas al norte, lo cual rememoraría a nivel simbólico, la organización espacio-temporal de la capital de los incas (Hyslop 1985; Hyslop y Schobinger 1991; Farrington 1998, 2014).

Este enclave inca debió entonces representar un indicador de límite de espacio y tiempo dentro del Tawantinsuyu en su proceso expansivo hacia el llano tucumano. En primer lugar, incorporando al sistema de huacas estatales el conjunto de cumbres del Aconquija al poniente, y segundo, controlando de manera indirecta los espacios de yunga al oriente, como refiere la toponimia a los pies del Aconqui- 
ja, p. e. Alpachiri, la Cocha y Rumi Punco, al sur de Tucumán. Esto habría permitido a los incas incorporar los territorios de las actuales provincias de Tucumán y Santiago del Estero, gracias a la prolongación de las esferas de interacción que ya mantenía con las jefaturas políticas de los valles Calchaquíes en Catamarca.

Se han retomado planteamientos de Farrington (2014), Moyano (2010b, 2013), Moyano y Díaz (2013), Pino (2004, 2005) y Zuidema $(1980,1989,2011)$, para plantear que la existencia del ushnu en La Ciudacita, además de funciones políticas y administrativas, vinculadas con el espacio de frontera, cumplió con ser un lugar la observación del cielo en tiempos de los incas. Más aún, suponemos que también habría servido como un centro para un sistema local de ceques o líneas proyectadas al horizonte a partir de referentes astronómicos y sitios sagrados en algunas cumbres locales, gracias a la sacra- lización y resignificación de los espacios conquistados. Esto confiere al sitio características casi únicas, posicionándolo, junto con el ushnu de El Apunao y el gnomon de Uña Tambo en los Nevados de Cachi, provincia de Salta (Jacob et al. 2011, 2013), como uno de los observatorios astronómicos situado a mayor altitud al sur del Cuzco.

Siguiendo los planteamientos de Raffino (2004), creemos que la arquitectura en La Ciudacita, en particular su ushnu, habrían actuado al igual que en otros territorios conquistados por el imperio, como elementos de dominación ideológica asociados a recursos minerales, montañas y cuerpos de agua. La ubicación geográfica del sitio le confiere una posición que permite la articulación de ambientes tan diferenciados como los valles mesotermales y las llanuras orientales, así como la interacción entre los grupos sociales que se emplazaron en

\section{Carta luni-solar La Ciudacita (ushnu)}

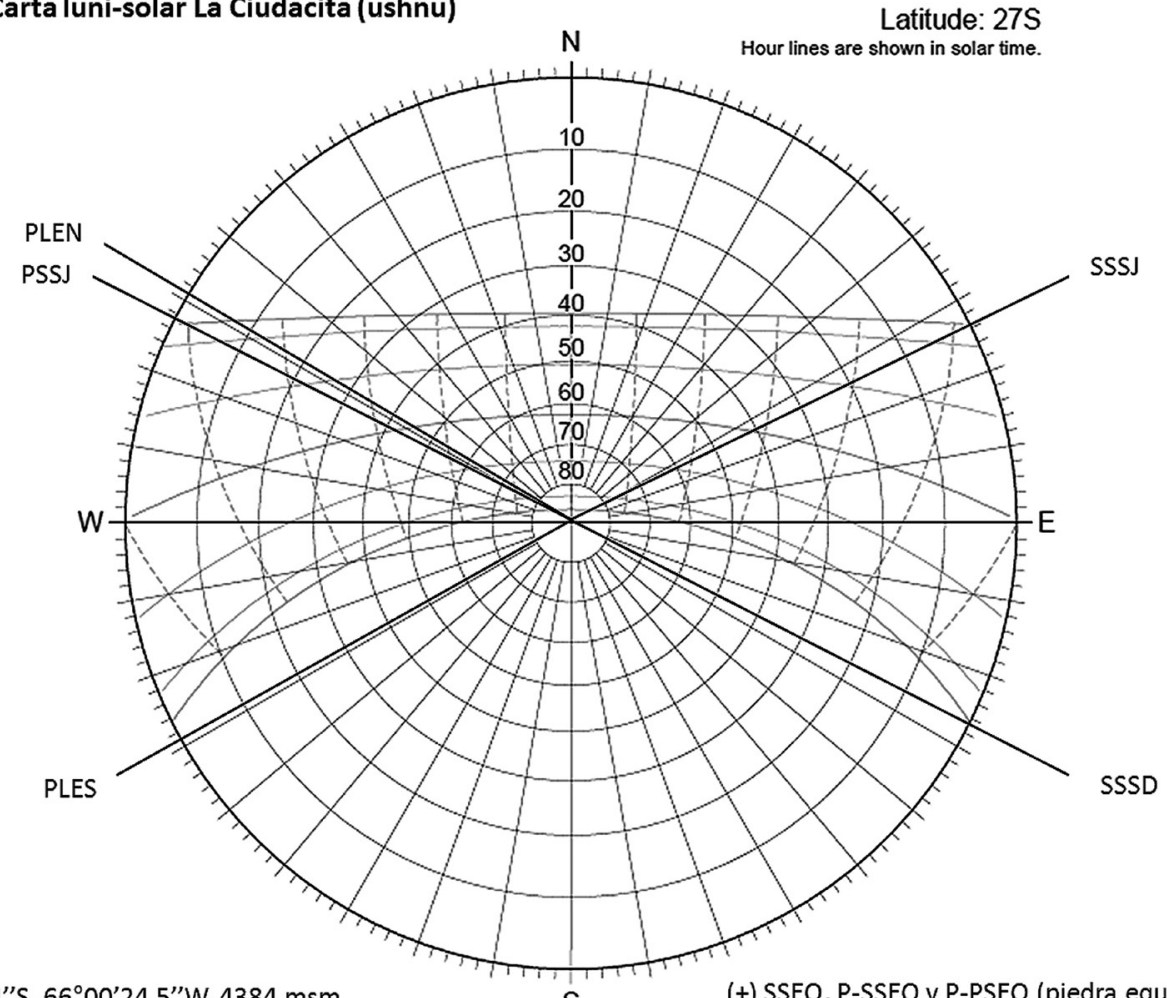

$27^{\circ} 10^{\prime} 52.4^{\prime \prime} \mathrm{S}, 66^{\circ} 00^{\prime} 24.5^{\prime \prime} \mathrm{W}, 4384 \mathrm{msm}$

(+) SSEQ, P-SSEQ y P-PSEQ (piedra equinoccial)

Figura 15. Carta lunisolar La Ciudacita (Moyano 2013). 
los mismos; hablamos de articulación porque la hegemonía inca no intentó anular a los grupos locales, por el contrario, se apoyó en sus estructuras socioeconómicas (Rostworowski 1999). En términos más amplios, también pudo relacionarse con algunas estrategias políticas e ideológicas que buscaron la incorporación de los cerros huacas a la religión estatal, gracias al uso de la memoria colectiva y las prácticas sociales, ligadas al paisaje y al origen mítico de cada población. En términos culturales, igualmente se relacionaba con el uso social del cielo (Iwaniszewski 2011), para reafirmar el status y las jerarquías sociopolíticas, las cuales ligaban a las elites locales con el Inca y las deidades celestes (Moyano 2013).

Orientaciones astronómicas hacia los lunisticios (paradas mayores al norte y sur), dejan la posibilidad abierta a que los incas conocieran la posición del nodo y con ello el conocimiento del momento de peligro de eclipses, gracias a la observación de la luna en ciclos cercanos a 18 y 19 años, cuya utilidad se relaciona con la posibilidad de conocer un momento de mal augurio, como el eclipse, dentro de la planificación coordinada del proceso expansivo y conquista en las provincias del llano, todavía no anexadas al Tawantinsuyu. De igual manera, se constató la orientación del vano de acceso a la plaza principal hacia la salida del sol en el solsticio de diciembre (SSSD) y una de la esquinas de la misma hacia la salida del sol en el solsticio de junio (SSSJ) (Bravo 1993; Beorchia 2011), lo que sumado a la utilización de un gnomon equinoccial en Pueblo Viejo Arriba, habría permitido conocer el fenómeno del crossover (P-SSEQ), y con ello calibrar la cuenta de 12013 meses lunares dentro del año solar de 365 días (Moyano 2013) (Figura 15).

En esta línea de trabajo, la evidencia etnohistórica del padre Diego Rodríguez de Figueroa (1910 [1565]) con respecto a los eclipses señala la importancia ritual de los mismos, al ser concebidos como un momento fatídico dentro del orden cosmológico de los incas (citado en Ziólkowski y Lebeuf 1993). De allí la necesidad de predecir los eclipses gracias al uso del ciclo metónico (19 años o 235 lunaciones), menos la cuenta de 12 meses lunares sinódicos, igual a I ciclo de Saros (18,03 años o 223 lunaciones).

En el caso de La Ciudacita, resultaría evidente a partir de la existencia de marcadores de horizonte y la utilización de la arquitectura para determinar la posición de la luna en los lunisticios y momentos cercanos al equinoccio de septiembre. Ese sistema de cuentas habría funcionado como una matriz interpretativa con base en la observación de la naturaleza, que intentó amortiguar la incertidumbre social y con ello, organizar determinadas actividades dentro del calendario a partir de una mnemotecnia lunar vinculada con la alegoría del nacimiento, plenitud y muerte de un astro en tiempos prehispánicos (Moyano 2012, 2013). En este sentido, el ushnu de la Ciudacita - dentro el proceso expansivo inca- puede ser visto como la materialización del orden espacio-temporal cuzqueño, además de un referente para la utilización de todos los elementos potencialmente sagrados de la topografía o entorno: cielo y tierra, como espacio completo (Bustamante y Moyano 2013; Jacob et al. 2013). Se contribuía con ello a la producción y reproducción de las estrategias de poder y a la construcción de distintas relaciones de reciprocidad, entre lo foráneo y lo local, lo sagrado y lo profano, y sobre todo a la justificación de un nuevo orden imperante o cosmovisión.

La arquitectura, entonces, actuará como un indicador en tiempos prehispánicos, p. e. la gran plaza y el ushnu (Pueblo Viejo de Abajo), no sólo en el sentido estrictamente funcional, sino también como referente espaciotemporal de distintas actividades de importancia ritual y calendárica en un espacio de frontera. Con la arquitectura los incas intentaron plasmar aspectos puntales de su cosmovisión, p. e. la división en tres mundos, la orientación al este, el culto a los antepasados y elementos celestes, etc., marcando con ello un dominio territorial y simbólico a lo largo del Tawantinsuyu, distanciándose de otras sociedades, con las que compartían no sólo un espacio común, sino también necesidades básicas derivadas del habitar a más de 4000 msnm (Ceruti 1999; Nielsen y Walker 1999; Acuto 1999, 2011, 2012; Leibowicz 2007, 2012; Moyano y Uribe 2012, entre otros). De esta forma los incas, a medida que extendían los límites del Tawantinsuyu, no sólo debieron movilizar tropas, bienes y mano de obra, sino ante todo un capital cultural que incluyó la religión y la observación del cielo - particularmente el seguimiento de los ciclos de la luna - como articuladores de una diplomacia andina destinada a conceptualizar y resignificar nuevos "espacios y tiempos" sagrados en los confines surorientales del Collasuyu. 
Agradecimientos A la Dirección de Parques Nacionales de Argentina, al Laboratorio de Geoarqueología de la Facultad de Ciencias Naturales e IML de la Universidad Nacional de Tucumán, a la Escuela Nacional de Antropología e Historia y su sistema de Becas Mixtas CONACYT y al sistema de Becas Posdoctorales del Instituto de Investigaciones Históricas de la UNAM, México. A los investigadores: Stanislaw Iwaniszewski, Ian Farrington, R. Tom Zuidema, Johanna Broda, Jorge Ianiszewski y Álvaro Martel por proporcionar material inédito de sus investigaciones. A Patricio Bustamante, Daniela Bustamante, Cristian Jacob e Iván Leibowicz por sus comentarios y la edición final del manuscrito. En especial a nuestras familias, colegas y amigos de montaña.

\section{* Referencias Citadas}

ACUTO, F. 1999. Paisaje y dominación: La constitución del espacio social en el Imperio Inka. En Sed Non Satiata. Teoría Social en la Arqueología Latinoamericana Contemporánea, A. Zarankin y F.A. Acuto (Eds.), pp. 33-75. Ediciones del Tridente, Buenos Aires.

2011. Encuentros coloniales, heterodoxia y ortodoxia en el valle Calchaquí norte bajo el dominio Inka. Estudios Atacameños 42:5-32.

2012. Landscapes of inequality, spectacle and control: Inka social order in provincial contexts. Revista Chilena de Antropología 25: 9-64.

ARRIAGA, P. J. de. 1964 [1621]. Extirpación de la idolatría del Pirú. En F. Esteve Barba (ed.), Crónicas peruanas de interés indígena, 191-277, Biblioteca de Autores Españoles 209, Madrid.

ATAliVA, V., A. MARTEL, C. SOMONTE y S. LÓPEZ. 2010. Notas marginales desde el sitio incaico Nevados del Aconquija (Tucumán, Argentina).Andes 21: 161-186.

AVENI, A. 2005. Observadores del cielo en el México antiguo. Fondo de Cultura Económica, México, D.F.

BAZAN, A. 2004. Ciudades y sitios Inka del territorio Catamarqueño. En El Shincal de Quimivíl, R. Raffino (Ed.), prólogo. Editorial Sarquís, San Fernando del valle de Catamarca.

BAUER, B. y D. DEARBORN. 1998. Astronomía e imperio en los Andes. Centro de Estudios Regionales Andinos Bartolomé de Las Casas, Cuzco.

BELMONTE, J. 1999. Las leyes del cielo, astronomía y civilizaciones antiguas. Ediciones Temas de Hoy, Madrid.

BERTRÁN DE QUINTANA, M. 1982. El Sol en la Mano. Universidad Nacional Autónoma de México, México, D.F.
BEORCHIA, A. 1985. La fabulosa ciudad perdida de los Nevados de Aconquija. CIADAM 5: 57-65.

2001. Astronomía indiana. Revista del C.I.A.D.A.M. 6: 195214 .

BRAVO, O. 1993. El Enigma de la Ciudacita. Arqueoastronomía de los Nevados del Aconquija, Provincia de Tucumán. Revista CET 3:5-14.

BUSTAMANTE, P. 2006. Hierofanía y pareidolia como propuestas de explicación parcial, a la sacralización de ciertos sitios, por algunas culturas precolombinas de Chile. Rupestreweb, http://rupestreweb.info/hierofania.html

2007. Pareidolia y apofenia como fenómenos extendidos y como herramienta de análisis de obras rupestres y sitios arqueológicos pertenecientes a diversas culturas. Rupestreweb, http:// www.rupestreweb.info/pareidoliaz.html

2008. Posible ubicuidad espacio-temporal de la triada pareidolia - apofenia - hierofanía, como probable origen de la sacralización de algunos elementos del paisaje. Rupestreweb, http:// www.rupestreweb.info/triada.html

BUSTAMANTE, P. y R. MOYANO. 2013. Cerro Wangüelen: obras rupestres, observatorio astronómico-orográfico Mapuche-Inca y el sistema de ceques de la cuenca de Santiago. Rupestreweb (en prensa).

CERUTI, C. 1999. Cumbres Sagradas del Noroeste Argentino. Editorial Universitaria de Buenos Aires, EUDEBA, Buenos Aires.

COBO, B. 1892 [1653]. Historia del Nuevo Mundo. Sociedad de Bibliófos Andaluces; notas e ilustraciones de D. Marcos Jiménez de la Espada, Imp. De E. Rasco, Sevilla. https://archive.org/details/ historiadelnuevooandagoog 
DA SILVA, C. 2004. The spring full Moon. JHA XXXV: 475-478.

2010. Neolithic cosmology: the equinox and the spring full Moon. Journal of Cosmology 9: 2207-2216.

DILLEHAY, T. y P. NETHERLY. 1998. La frontera del estado Inca, T. Dillehay y P. Netherly (Comps.). Fundación Alexander von Humboldt, Editorial Abya-Yala, Quito.

FARRINGTON, I. 1998. The concept of Cusco. Tawantinsuyu 5: 5359.

2014. The Centre of the World and the Cusco usnu complexes. En Inca Sacred Space. Landscape, Site and Symbol in the Andes, F. Meddens, C. McEwan, K. Willis y N. Branch (Eds.), pp. 197207. Archetype Publications, London.

GASPARINI, G. y L. MARGOLIES. 1977. Arquitectura Inka. Centro de Investigaciones Históricas y Estéticas, Facultad de Arquitectura y Urbanismo, Universidad Central de Venezuela, Caracas.

GIDDENS, A. 1995. La constitución de la sociedad. Bases para la teoría de la estructuración. Amorrortu Editores, Buenos Aires.

GONZÁLEZ, A. R. 1980. Patrones de asentamiento incaico en una provincia marginal del Imperio. Implicancias socio-culturales. Relaciones de la Sociedad Argentina de Antropología XIV (1): 63-82.

GONZÁLEZ, L. y M. TARRAGÓ. 2005. Vientos del sur. El valle de Yocavil (Noroeste Argentino) bajo la dominación incaica. Estudios Atacameños 29: 67-95.

GREEN, R. 1999. Spherical astronomy. Cambridge University Press, New York.

HARDMAN, C. JR. y M.H. HARDMAN. 1992. Linear solar observatory theory: The development of concepts of time and calendar. North American Archaeologist 13 (2): 149-172.

HYSLOP, J. 1985. Inkawasi, the New Cusco. En British Archaeological Reports, International Series 234: XII-147.

1990. Inka settlement planning. University of Texas Press, Austin.

HYSLOP, J. y J. SCHOBINGER. 1991. Las ruinas incaicas de los Nevados del Aconquija. Comechingonia Año 9: 15-30.

HAWKINS, G. 1966. Astro-archaeology. Smithsonian Institution, Astrophysical Observatory, Cambridge.

IANISZEWSKI, J. 2010. Guía a los cielos australes: astronomía básica para el hemisferio sur. Editorial Mitra, Santiago de Chile.
IWANISZEWSKI, S. 2011. The sky as a social field. En Archaeoastronomy and ethnoastronomy: Building bridges between cultures, C. Ruggles (Ed.), pp. 30-37. Cambridge University Press, Cambridge.

JACOB, C., R. MOYANO, F. ACUTO e I. LEIBOWICZ. 2011. Quilca del cielo: valle Calchaquí, Salta, Argentina. Boletín APAR 4 (10): 348-350.

JACOB, C., R. MOYANO e I. LEIBOWICZ. 2013. Está saliendo el Sol. La Intihuatana del sitio Uña Tambo, Nevados de Cachi, Salta, Argentina. Ponencia presentada en Simposio 24 (Tawantinsuyu 2013), XVIII CNAA, La Rioja.

KING, D. 1993. Folk astronomy in the service of religion: the case of Islam. En Astronomies and Cultures, C. Ruggles y N.J. Saunders (Eds.), pp. 124-138. University of Colorado Press, Niwot, Colorado.

LAZAROVICH, M. 1996. Informe del Primer Viaje de Reconocimiento al Parque Nacional Campo de los Alisos. Administración de Parques Nacionales (APN), Delegación Técnica regional del Noroeste, Salta.

LEBEUF, A. 2003. Les eclipses dans l'ancien Mexique. Jagiellonian University Press, Kraków.

Leibowicz, I. 2007. Espacios de poder en La Huerta, Quebrada de Humahuaca. Estudios Atacameños. Arqueología y Antropología Surandinas 34: 51-70.

2012. Ideología y Espacio: Conquista Inka en la Quebrada de Humahuaca, Jujuy, Argentina. Revista Chilena de Antropología 25: 65-91.

LORANDI, A. 1998. Los diaguitas y el Tawantinsuyu: una hipótesis de conflicto. En La frontera del estado Inca, pp. 197-214. Fundación Alexander von Humboldt y Editorial Abya-Yala, Quito.

MANSFELD, F. 1948. La ciudad legendaria del Aconquija. Revista Geográfica Americana XXIX: 53-59.

MARSHACK, A. 1972. The Roots of Civilization: the Cognitive Beginning of Man's First Art, Symbol and Notation. McGraw-Hill, New York.

MARTEL, A., V. ATALIVA, C. SOMONTE y S. LÓPEZ. 2002. Informe sobre la prospección arqueológica y evaluación del estado de los recursos culturales del sitio Nevados de Aconquija, Parque Nacional Campo de los Alisos. Consejo Nacional de Investigaciones Científicas y Técnicas (CONICET), Buenos Aires.

MARTZ DE LA VEGA, H., R. MOYANO, S. IWANISZEWSKI y M. PÉREZ NEGRETE. 2013. Hansómetro. Programa libre para cómputo de arqueoastronomía en Excel. En constante actualización. Escuela Nacional de Antropología e Historia (ENAH), México. 
McCLUSKEY, S. 1986. Lunar astronomies of the western Pueblos. Ponencia presentada en Oxford II International Conference on Archaeoastronomy, Mérida.

MEDDENS, F. 1997. Function and meaning of the usnu in the late horizon Peru. Tawantinsuyu 3: 5-14.

MEEUS, J. 2002. The harvest Moon. En More mathematical astronomy. Willmann-Bell, Richmond, Virginia.

MEEUS, J. y D. SAVOIE. 1992. The history of the tropical year. Journal of the British Astronomical Association 102 (1): 40-42.

MOESGAARD, K. 1980. The full Moon serpent. A foundation stone of ancient astronomy? Centaurus 24: 51-96.

MONTGOMERY, S. 1999. The Moon and the western imagination. University of Arizona Press, Tucson.

MOYANO, R. 2010a. La mano de dios en Socaire: estudio de un calendario agrícola en Atacama, norte de Chile. Tesis para optar al grado de maestro en arqueología. Escuela Nacional de Antropología e Historia. México, D.F.

2010b. El ushnu y la astronomía de horizonte en Viña del Cerro. Chungara Revista de Antropología Chilena 42 (2): 419-432.

2011. Sub-tropical astronomy in southern Andes: the ceque system in Socaire, Atacama, northern Chile. EnArchaeoastronomy and ethnoastronomy: Building bridges between cultures, C. Ruggles (Ed.), pp. 93-105. Cambridge University Press, Cambridge.

2012. La Luna como objeto liminal en la concepción del tiempo indicativo entre los incas. Revista Haucaypata. Investigaciones arqueológicas del Tahuantinsuyo 4: 6-16.

2013. La Luna como objeto de estudio antropológico: el ushnu y la predicción de eclipses en contextos incas del Collasuyu. Tesis para optar al grado de doctor en arqueología. Escuela Nacional de Antropología e Historia. México, D.F.

MOYANO, R. y C. URIBE. 2012. El volcán Chiliques y el "moraren-el-mundo" de una comunidad atacameña del norte de Chile. Estudios Atacameños 43: 187-208.

MOYANO, R. y G. DÍAZ. 2013. Astronomía lunar en los nevados de Aconquija: el ushnu y la predicción de eclipses en los Andes del Collasuyu. Ponencia presentada en Mesa de Comunicaciones del NOA, ХVIII CNAA, La Rioja.

NIELSEN, A., W.H. WALKER. 1999. Conquista ritual y dominación política en el Tawantinsuyu. El caso de Los Amarillos. Sed Non Satiata. Teoría Social en la Arqueología Latinoamericana Contem- poránea, A. Zarankin y F. Acuto (Eds.). Ediciones del Tridente, Buenos Aires.

NOLI, E. 2012. Indios Ladinos, Criollos Aindiados. Procesos de Mestizajey Memoria Étnica en Tucumán. Prohistoria Ediciones, Rosario.

PINO, J. 2004. El ushnu Inka y la organización del espacio en los principales tampus de los wamani de la sierra central del Chinchaysuyu. Chungara. Revista de antropología chilena 36 (2): 303-311.

2005. El ushnu y la organización espacial astronómica en la sierra central del Chinchaysuyu. Estudios Atacameños 29: 143-161.

PAULOTTI, O. 1959. La ruinas de los Nevados del Aconquija, noticia preliminar. Runa 9 (1-2): 126-136.

1967. Las ruinas de los Nevados del Aconquija. Runa 10 (1-2): 354-370.

RAFFINO, R. 1981. Los Inkas del Kollasuyu. Ramos Americana Editores, Buenos Aires.

2004. El Shincal de Quimivíl. Editorial Sarquís, San Fernando del valle de Catamarca.

RAFFINO, R., D. GOBBO, R. VÁZQUEZ, A. CAPPARELLI, V. MONTES, R. ITURRIZA, C. DESCHAMPS y M. MANNASERO. 1997. El ushnu de El Shincal de Quimivil. Tawantinsuyu 3: $22-39$.

ROSTWOROWSKI, M. 1999. Historia del Tawantinsuyu, za ed. Instituto de Estudios Peruanos, Lima.

SAMPIETRO, M. M., L. NEDER, J. ROLDÁN y M. VATTUONE. 2008. Mother earth: soil and people relationships during the prehispanic period (northwest Argentina). World Archaeology 40 (2): 190-205.

SANHUEZA, CECILIA. 2008. Territorios, prácticas rituales y demarcación del espacio en Tarapacá en el siglo XVI. Boletín del Museo Chileno de Arte Precolombino 13 (2): 57-75.

SCATTOLIN, M.C. y M.A. KORSTANJE. 1994. Tránsito y frontera en los Nevados del Aconquija. Revista Arqueología 4: 165-197.

SCHAEFER, B. 1992. The length of the lunar month. Archaeoastronomy 17:32-42.

SCHOBINGER, J. y MA. C. CERUTI. 20o1. Arqueología de alta montaña en los Andes argentinos. En Historia prehispánica argentina, E. Berberián y A. Nielsen (Eds.), tomo II, pp. 523-559. Editorial Brujas, Buenos Aires. 
SILVA, F. y F. PIMIENTA. 2012. The crossover of the Sun and the Moon. JHA XLIII: 191-208.

SPRAJC, I. 2010. Propiedades astronómicas de la arquitectura prehispánica en la isla de Cozumel, Quintana Roo, México. En Los Investigadores de la Cultura Maya 18 (II), pp. 113-136. Universidad Autónoma de Campeche, Campeche.

STEELE, J. 2000. Observation and Predictions of Eclipse Times by Early Astronomers, Volume 4. Kluwer Academic Publishers. Dordrecht, Boston, London.

STERN, S. 2008. The Babylonian month and the new Moon: sighting and prediction. JHA XXXIX: 19-42.

STUVEN, H. 1972. 42 Gráficos solares para ciudades de Chile y Argentina. Facultad de Arquitectura, Universidad de Chile, Santiago de Chile.

TILLEY, C. 1994. A phenomenology of landscape: Places, paths and monuments. Oxford, Berg.

WILLIAMS, V. 2000. El imperio Inka en la provincia de Catamarca. Intersecciones en Antropología 1: 55-78.

WILLIAMS, V., C. SANTORO, A. ROMERO, J. GORDILLO, D. VALENZUELA y V. STANDEN. 2009. Dominación Inca en los valles occidentales (sur del Perú y norte de Chile). Andes 7: 615-654.

WÜRSCHMIDT, E. 1952. Las Ruinas de las Pavas, en los Nevados del Aconquija. Descripción Arqueo-Geográfica. Revista Llokhana 1.

ZIÓLKOWSKI, M. y A. LEBEUF. 1993. Were the Incas able to predict lunar eclipses? En Archaeoastronomy in the 1990s, C. Ruggles (Ed.), pp. 298-308. Group D. Publications, Loughborough Leicestershire.

ZUIDEMA, R.T. 1980. El ushnu. Revista de la Universidad Complutense 28: $317-362$.

1982. Catachillay: The role of the Pleiades and of the Southern Cross, and Alpha and Beta Centauri in the Calendar of the Incas. En Ethnoastronomy and archaeoastronomy in the America Tropics, A. Aveni y G. Urton (Eds.), 385: 203-229. Annals of the New York Academy of Sciences, New York.

1989. Reyes y guerreros: Ensayos de la cultura andina. FOMCIENCIAS, Lima.

2011. El calendario Inca: Tiempo y espacio en la organización ritual del Cusco; La idea del pasado. Fondo Editorial del Congreso del Perú y Pontificia Universidad Católica del Perú, Lima. 